\title{
Complex Quantum Group, Dual Algebra and Bicovariant Differential Calculus
}

\author{
Ursula Carow-Watamura ${ }^{1}$ and Satoshi Watamura ${ }^{2}$ \\ 1 Department of Physics, Faculty of Science, Tohoku University, Sendai 980, Japan \\ 2 Department of Physics, College of General Education, Tohoku University, Sendai 980, Japan
}

Received January 11, 1992; in revised form July 15, 1992

\begin{abstract}
The method used to construct the bicovariant bimodule in ref. [CSWW] is applied to examine the structure of the dual algebra and the bicovariant differential calculus of the complex quantum group. The complex quantum group $\operatorname{Fun}_{q}(S L(N, C))$ is defined by requiring that it contains $\operatorname{Fun}_{q}(S U(N))$ as a subalgebra analogously to the quantum Lorentz group. Analyzing the properties of the fundamental bimodule, we show that the dual algebra has the structure of the twisted product $\operatorname{Fun}_{q}(S U(N)) \widetilde{\otimes} \operatorname{Fun}_{q}(S U(N))_{\text {reg. }}^{*}$. Then the bicovariant differential calculi on the complex quantum group are constructed.
\end{abstract}

\section{Introduction}

The question of whether the physics of microscopic scale such as the Planck scale is incorporated by the noncommutativity, which is different from the one provided by the quantization of the field theory, has concerned physicists already for a long time.

Such a theory may be described by a noncommutative algebra belonging to a wider class than the one which physicists are now handling. To consider such a possibility, we need to understand more about the general structure of the noncommutative algebra. From this point of view, the quantum group, a class of noncommutative Hopf algebra found in the investigation of the integrable systems is a very interesting example [Dri, Jim, Wor1]. Imposing the covariance under the quantum group, we can also get some other examples of the noncommutative algebra such as the algebra of the comodule, i.e. the quantum space (quantum plane) introduced by Manin [Manin, RTF]. These algebras give some interesting examples of noncommutative algebras such as the quantum Lorentz group $[\mathrm{PW}, \mathrm{CSSW}]$ and the quantum Poincare group [LNRT, LNR]. The construction of the noncommutative differential calculus on the quantum group [Wor2, Rosso, Stach, Jur, Weich, MNW, MH, CSWW] and also on the quantum space [Wess, WZ,Zumino, Pusz, CSW, Schm] shows us various promising features peculiar to the noncommutative algebra. 
In this paper we consider another class of noncommutative algebra which is also related to the quantum group: the algebra of the bicovariant bimodules generated by the fundamental bicovariant bimodule. We have used such an algebra implicitly in our previous paper [CSWW], where we have investigated the bicovariant bimodule over the compact quantum groups in order to write down the explicit formulae of the differential calculus proposed by Woronowicz [Wor2]. Here we consider the bicovariant bimodule over the complex quantum group $\operatorname{Fun}_{q}(S L(N, C))$.

To define the commutation relations of the generators of the complex quantum group $\operatorname{Fun}_{q}(S L(N, C))$, we can use the same method as applied in the construction of the quantum Lorentz group [PW,CSSW]. The problem of defining the quantum Lorentz group, i.e. the $*$-Hopf algebra $\operatorname{Fun}_{q}(S L(2, C))$ was to find the commutation relations between the generators $M_{j}^{i}$ and $\left(M_{j}^{i *}\right)$. The result in ref. [CSSW] can be generalized straightforwardly to obtain the commutation relations of $\operatorname{Fun}_{q}(S L(N, C))$.

Here we analyze the structure of the bicovariant bimodule over the complex quantum group $\operatorname{Fun}_{q}(S L(N, C))$. We consider the algebra of the bicovariant bimodule generated by the right invariant basis requiring the covariance under the coaction. We define the bicovariant commutation relations among the right invariant basis and we find the relation among the bimodules which have the different ordering of the product of the right invariant basis. The commutation relations among the bimodules then can be translated into the commutation relations among the functionals where the product is given by the convolution product. Using this result we give the definition of the dual algebra of the complex quantum group $\operatorname{Fun}_{q}(S L(N, C)) .{ }^{1}$ We show that the resulting dual algebra of the complex quantum group has the structure of the twisted product $\operatorname{Fun}_{q}(S U(N)) \tilde{\otimes} \operatorname{Fun}_{q}(S U(N))_{\text {reg }}^{*}$. Using these results we construct the bicovariant differential calculus on the complex quantum group. Comparing with the compact quantum group case, we find that the condition of the nilpotency of the exterior derivative plays a crucial role.

This paper is organized as follows. In Sect. 1 we introduce the definition of the $\operatorname{Fun}_{q}(S L(N, C))$. In Sect. 2, the bicovariant bimodule is defined and the functionals relating the left and right multiplication are calculated in terms of the $\hat{\mathbf{R}}$-matrix of $\operatorname{Fun}_{q}(S U(N))$. In Sect. 3, we examine the structure of the product of the bicovariant bimodules defining the commutation relation between the fundamental bimodules. In Sect. 4, we translate the result obtained in Sect. 3 into the commutation relations among the functionals and define the dual algebra of $\operatorname{Fun}_{q}(\operatorname{SL}(N, C))$. In Sect. 5, using the bicovariant bimodule possible differential calculi on complex quantum groups are proposed.

\section{Complex Quantum Group}

Let us introduce briefly the concept and notations which we need in the following. The quantum group $\operatorname{Fun}_{q}(S L(N, C))$ is defined as a *-Hopf algebra of the

\footnotetext{
${ }^{1}$ In ref. [DSWZ], the universal enveloping algebra of the complex quantum group is also derived by generalizing the methods of RTF [RTF]. Relating investigation about the quantum Lorentz algebra, i.e., the dual algebra of $\operatorname{Fun}_{q}(S L(2, C))$ have been performed by [SWZ, OSWZ]
} 
$q$-deformed Fun $(S L(N, C))$. The generators of the quantum group are the matrix elements $M_{j}^{i}$ of the $N \times N q$-fundamental representation and its $*$-conjugate where the $*$-operation is the antimultiplicative involution. The coproduct $\Delta$, counit $\varepsilon$ and antipode $\kappa$ are defined in the standard way [Abe, Wor1]. (For more details of our notations see ref. [CSWW].)

$$
\begin{aligned}
& \Delta\left(M_{j}^{i}\right)=M_{i^{\prime}}^{i} \otimes M_{j}^{i^{\prime}}, \\
& \Delta\left(\bar{M}_{j}^{i}\right)=\bar{M}_{j}^{i^{\prime}} \otimes \bar{M}_{i^{\prime}}^{i},
\end{aligned}
$$

and

$$
\varepsilon\left(M_{j}^{i}\right)=\varepsilon\left(\bar{M}_{j}^{i}\right)=\delta_{j}^{i},
$$

where we have introduced $\bar{M}$ as

$$
\bar{M}_{j}^{i} \equiv\left(M_{i}^{j}\right)^{*} .
$$

The complex quantum group $\operatorname{Fun}_{q}(S L(N, C))$ is a noncommutative algebra and the commutation relations among the generators are given by the matrix $\hat{\mathbf{R}}_{k l}^{i j}$ of $\operatorname{Fun}_{q}(S U(N))$ which satisfies the braid equation:

$$
\hat{\mathbf{R}}_{12} \hat{\mathbf{R}}_{23} \hat{\mathbf{R}}_{12}=\hat{\mathbf{R}}_{23} \hat{\mathbf{R}}_{12} \hat{\mathbf{R}}_{23} \text {. }
$$

With the $\hat{\mathbf{R}}$-matrix, the commutation relations can be represented as:

$$
\begin{aligned}
\hat{\mathbf{R}}_{i^{\prime} j^{\prime}}^{i j} M_{k}^{i^{\prime}} M_{l}^{j^{\prime}} & =M_{i^{\prime}}^{i} M_{j^{\prime}}^{j} \hat{\mathbf{R}}_{k l}^{i^{\prime} j^{\prime}}, \\
\hat{\mathbf{R}}_{i j}^{i^{\prime} j^{\prime}} \bar{M}_{j^{\prime}}^{l} \bar{M}_{i^{\prime}}^{k} & =\bar{M}_{j}^{j^{\prime}} \bar{M}_{i}^{i^{\prime}} \hat{\mathbf{R}}_{i^{\prime} j^{\prime}}^{k l} .
\end{aligned}
$$

The second relation (1.7) is just the $*$-conjugation of (1.6). Note that we take the normalization of the $\hat{\mathbf{R}}$-matrix as

$$
\hat{\mathbf{R}}=q^{-1 / N}\left(q \mathscr{P}_{S}-q^{-1} \mathscr{P}_{A}\right),
$$

where $\mathscr{P}_{S}$ and $\mathscr{P}_{A}$ are the $q$-deformed projectors onto the $q$-symmetric and $q$-antisymmetric product, respectively (see also ref. [CSWW]).

To complete the definition of the quantum group $\operatorname{Fun}_{q}(\operatorname{SL}(N, C))$, we must also give the commutation relation among $M$ and $M^{*}$. In this paper, we define it such that the resulting algebra reduces under the unitarity condition to the compact quantum group $\operatorname{Fun}_{q}(S U(N))$ like in the case of the quantum Lorentz group $[\mathrm{PW}, \mathrm{CSSW}]$ and thus we can take $q>0 .^{2}$

A heuristic argument to find this relation is the following: Multiplying the antipode on $M$ we can rewrite Eq. (1.6) as

$$
\hat{\mathbf{R}}_{k l l}^{i j} \kappa\left(M_{i}^{s}\right) M_{k}^{k^{\prime}}=M_{j^{\prime}}^{j} \hat{\mathbf{R}}_{k t}^{s j^{\prime}} \kappa\left(M_{l}^{t}\right) .
$$

Recalling that the unitarity condition in the $\operatorname{Fun}_{q}(S U(N))$ is given by $\bar{M}=\kappa(M)$, we replace $\kappa(M)$ in Eq. (1.9) by $\bar{M}$. Then, we get

$$
\bar{M}_{i^{\prime}}^{i} M_{k}^{j^{\prime}} \hat{\mathbf{R}}_{j^{\prime} l}^{-1 i^{\prime} j}=\hat{\mathbf{R}}_{k i^{\prime}}^{-1 i j^{\prime}} M_{j^{\prime}}^{j} \bar{M}_{l}^{i^{\prime}} .
$$

We take this relation as the definition of the commutation relation among the $M$ and $\bar{M}$ in the complex quantum group. By construction it consistently reduces to the $\operatorname{Fun}_{q}(S U(N))$ when we impose the unitarity condition.

\footnotetext{
${ }^{2}$ The complex quantum groups of this type are also proposed by Podleś [Pod1]. The commutator in Eq. (1.10) is also considered by [Koor] and [DSWZ]
} 
Note that we can also consider the algebra which is defined by taking in Eq. (1.10) $\hat{\mathbf{R}}$ instead of $\hat{\mathbf{R}}^{-1}$ which leads to the equivalent algebra. The choice made in Eq. (1.10) is such that the conventions in this paper coincide with the ones of our previous papers.

The generators also satisfy

$$
\operatorname{det}_{q} M=1 \text { and }\left(\operatorname{det}_{q} M\right)^{*}=1 .
$$

The $q$-deformed determinant in Eq. (1.11) is defined by using the $N^{\text {th }}$ rank $q$-antisymmetric tensor

$$
\varepsilon_{i_{1} \ldots i_{N}}=\frac{q^{\frac{N(N-1)}{4}}}{\sqrt{\llbracket N-1 \rrbracket !}}(-q)^{l(\sigma)},
$$

where $\sigma$ denotes the permutation of the suffices $\left(i_{1}, \ldots, i_{N}\right)=\sigma(1,2, \ldots, N)$ and $l(\sigma)$ is the minimal number of inversions in the permutation $\sigma$ [Dri]. The $q$-number is defined as $\llbracket x \rrbracket=\frac{q^{x}-q^{-x}}{q-q^{-1}}$. We also define the tensor with upper indices as $\varepsilon^{i_{1} \ldots i_{N}}=(-1)^{N} \varepsilon_{i_{1}} \ldots i_{v}$. The $q$-determinant is then given by

$$
\varepsilon_{i_{1} \ldots i_{N}} M_{j_{1}}^{i_{1}} \ldots M_{j_{N}}^{i_{N}}=\operatorname{det}_{q} M \varepsilon_{j_{1} \ldots j_{N}} .
$$

With the normalization of determinant as (1.11), the antipode $\kappa$ is defined by

$$
\begin{aligned}
& \kappa\left(M_{j}^{i}\right)=\varepsilon^{i i_{1} . i_{N-1}} M_{i_{1}}^{j_{1}} \ldots M_{i_{N-1}}^{j_{N-1}} \varepsilon_{j_{1} \ldots j_{N-1} j}, \\
& \kappa\left(\bar{M}_{i}^{j}\right)=\varepsilon^{j j_{1} \ldots j_{N-1}} \bar{M}_{j_{N-1}}^{l_{N-1}} \ldots \bar{M}_{j_{1}}^{i_{1}} \varepsilon_{l_{1} \ldots i_{N-1} i},
\end{aligned}
$$

The complex quantum group $\mathscr{A}=\operatorname{Fun}_{q}(\operatorname{SL}(N, C))$ is defined as the $*$-Hopf algebra with

$$
\mathscr{A} \equiv \mathbf{C}\left\langle M_{j}^{i}, \bar{M}_{j}^{i}\right\rangle /((1.6),(1.7),(1.10),(1.11)) .
$$

Although these definitions are sufficient to describe the complex quantum group, it is convenient to introduce the following convention for raising and lowering indices: Using the antisymmetric tensor, we represent the lower (upper) suffix by $N-1$ antisymmetrized upper (lower) indices.

For the lower and upper suffix $V_{i}$ and $W^{j}$ :

$$
\begin{array}{ll}
V^{[i]} \equiv V_{j} \varepsilon^{j[i]}, & V_{i} \equiv V^{[j]} \varepsilon_{[j] i}, \\
W_{[i]} \equiv \varepsilon_{[i] j} W^{j}, & W^{i} \equiv \varepsilon^{i[j]} W_{[j]},
\end{array}
$$

where $[i]=\left[i_{1} \ldots i_{N-1}\right]$ denotes $N-1 q$-antisymmetrized indices as introduced in ref. [CSWW]. We have taken the normalization of the $\varepsilon$ tensor in Eq. (1.12) such that

$$
\varepsilon^{i[j]} \varepsilon_{[j] k}=\delta_{k}^{i}
$$

Using this convention we can write the same object either with usual index or with $N-1$ antisymmetrized indices. For example

$$
\bar{M}_{[j]}^{[i]} \equiv \varepsilon_{[j] k} \bar{M}_{l}^{k} \varepsilon^{l[i]} .
$$


The reason to introduce this notation is that then the coproduct of $\bar{M}$ becomes similar to the case of the generators $M_{j}^{i}$ :

$$
\Delta\left(\bar{M}_{[j]}^{[i]}\right)=\sum_{[k]} \bar{M}_{[k]}^{[i]} \otimes \bar{M}_{[j]}^{[k]},
$$

and the naturally ordered index structure is kept such that the graphical analysis explained in ref. [CSWW] can be applied without any complication. Actually, some of the equations in the following sections become simpler when we use the antisymmetrized suffix. In such a case, we give the equations in the simpler form and sometimes with using both types for clearness.

With this notation Eq. (1.7) and Eq. (1.10) are rewritten as

$$
\begin{gathered}
\hat{\mathbf{R}}_{\left[i^{\prime}\right]\left[j^{\prime}\right]}^{[i][j]} \bar{M}_{[k]}^{\left[i^{\prime}\right]} \bar{M}_{[l]}^{\left[j^{\prime}\right]}=\bar{M}_{\left[i^{\prime}\right]}^{[i]} \bar{M}_{\left[j^{\prime}\right]}^{[j]} \hat{\mathbf{R}}_{[k][l]}^{\left[i^{\prime}\right]\left[j^{\prime}\right]}, \\
\hat{\mathbf{R}}_{\left[j^{\prime}\right] i}^{i[j]}, \bar{M}_{[k]}^{\left[j^{\prime}\right]} M_{l}^{i^{\prime}}=\bar{M}_{i^{\prime}}^{i} M_{\left[j^{\prime}\right]}^{[j]} \hat{\mathbf{R}}_{[k] l}^{i^{\prime}\left[j^{\prime}\right]} .
\end{gathered}
$$

Note that we do not apply the rule of raising and lowering suffices (1.17) and (1.18) for the $\hat{\mathbf{R}}$-matrix. The definition of each $\hat{\mathbf{R}}$-matrix is given in the Appendix.

\section{Bicovariant Bimodule over the Complex Quantum Group}

To construct the bicovariant bimodule [Wor2] of the general tensor representation, we first investigate the structure of the fundamental bicovariant bimodules, i.e., the $N$-dimensional bicovariant bimodules as is performed in ref. [CSWW]. We denote the right invariant basis as $\eta^{i}$. The right and left coactions are defined as

$$
\begin{aligned}
& \Delta_{R}\left(\eta^{i}\right)=\eta^{i} \otimes \mathbf{I}, \\
& \Delta_{L}\left(\eta^{i}\right)=M_{j}^{i} \otimes \eta^{j},
\end{aligned}
$$

where $\mathbf{I}$ is the unit of $\mathscr{A}$.

We also have to consider the $*$-conjugation of this bimodule. We denote the *-conjugation of the basis by $\bar{\eta}_{i}$, where

$$
\bar{\eta}_{i} \equiv\left(\eta^{i}\right)^{*}
$$

The $*$-conjugation of a bimodule is given as follows [Wor2]: For any $\rho$ which is an element of the bimodule

$$
(a \rho b)^{*}=b^{*} \rho^{*} a^{*}
$$

where $a, b \in \mathscr{A}$.

Taking the $*$-conjugation of Eqs. (2.1) and (2.2) we get the relations

$$
\begin{aligned}
& \Delta_{R}\left(\bar{\eta}_{i}\right)=\bar{\eta}_{i} \otimes \mathbf{I}, \\
& \Delta_{L}\left(\bar{\eta}_{i}\right)=\bar{M}_{i}^{j} \otimes \bar{\eta}_{j} .
\end{aligned}
$$

The latter equation may be written as

$$
\Delta_{L}\left(\bar{\eta}^{[i]}\right)=\bar{M}_{[j]}^{[i]} \otimes \bar{\eta}^{[j]},
$$


where

$$
\bar{\eta}^{[i]}=\bar{\eta}_{j} \varepsilon^{j[i]} .
$$

To complete the definitions of the bicovariant bimodule over $\mathscr{A}$ we have to give the relations between the left and right multiplication.

The requirement of bicovariance fixes the general form of these relations like in the case of the compact quantum groups as shown in ref. [Wor2]. In general, the relation of left and right multiplication for each right invariant basis is described by a functional $f_{j}^{i} \in \mathscr{A}^{\prime}$ [In this stage we denote the space of the linear functionals by $\left.\mathscr{A}^{\prime}.\right]$ :

$$
f_{j}^{i}: \mathscr{A} \rightarrow \mathbf{C} .
$$

As we shall see later there are two different functionals for each basis $\eta^{i}$ and $\bar{\eta}_{i}$ like in the case of $\operatorname{Fun}_{q}(S U(N))$. We distinguish these functionals with a suffix + and - as $f_{ \pm}$. Correspondingly, we have to distinguish also the right invariant bases for which the relation between the left and right multiplication is given by different functionals. Therefore we also introduce for them the corresponding suffix as $\eta_{ \pm}^{i}$ and $\bar{\eta}_{ \pm i}$, where

$$
\bar{\eta}_{ \pm i} \equiv\left(\eta_{ \pm}^{i}\right)^{*}
$$

In $\operatorname{Fun}_{q}(S U(N))$, due to the unitarity condition, we end up with 2 types of $N$ dimensional representations. On the other hand, when we consider the $\operatorname{Fun}_{q}(S L(N, C))$, all 4 types must be considered.

For the present case, this means that there exist functionals $f_{ \pm j}^{i}$ and $\bar{f}_{ \pm j}^{i}$ for each right invariant basis $\eta_{ \pm}^{i}$ and $\bar{\eta}_{ \pm i}$, where the relation between the right and left multiplication is represented as: $\forall a \in \mathscr{A}$,

$$
\begin{array}{ll}
\eta_{ \pm}^{i} a=\left(a * f_{ \pm j}^{i}\right) \eta_{ \pm}^{j}, & a \eta_{ \pm}^{i}=\eta_{ \pm}^{j}\left(a * f_{ \pm j}^{i} \circ \kappa\right), \\
\bar{\eta}_{ \pm}^{[i]} a=\left(a * \bar{f}_{ \pm[j]}^{[i]}\right) \bar{\eta}_{ \pm}^{[j]}, & a \bar{\eta}_{ \pm}^{[i]}=\bar{\eta}_{ \pm}^{[j]}\left(a * \bar{f}_{ \pm[j]}^{[i]} \circ \kappa\right) .
\end{array}
$$

The relation between the functionals $\bar{f}_{ \pm[j]}^{[i]}$ and $\bar{f}_{ \pm j}^{i}$ is given by using the rule (1.17) and (1.18)

$$
\bar{f}_{ \pm[j]}^{[i]}=\varepsilon_{[j] k} \bar{f}_{ \pm l}^{k} \varepsilon^{l[i]},
$$

where in order to keep the summation convention of the suffix, we have introduced the $\bar{f}_{ \pm j}^{i}$ as

$$
\bar{f}_{ \pm j}^{i} \equiv\left(f_{ \pm i}^{j}\right)^{*} .
$$

The *-conjugation of the functional $f \in \mathscr{A}^{\prime}$ is defined as

$$
f^{*}(a) \equiv\left(f\left(\kappa\left(a^{*}\right)\right)\right)^{*} .
$$

Equation (2.10) is the one given in ref. [Wor2]. Equation (2.11) is the $*$-conjugation of Eq. (2.10). To prove that they are the $*$-conjugation of each other we have to show how the convolution product of an element of $\mathscr{A}$ with an element of $\mathscr{A}^{\prime}$ behaves under the $*$-conjugation.

Proposition 1. The *-operation on the convolution product $\forall a \in \mathscr{A}$ and $\forall f \in \mathscr{A}^{\prime}$ is

$$
(a * f)^{*}=\left(a^{*} * g * \circ \kappa\right) .
$$


Proof. We can prove this equivalence directly using the definition of convolution product. The 1.h.s. is

$$
\begin{aligned}
(a * f)^{*} & =((f \otimes \mathrm{id}) \Delta(a))^{*} \\
& =\left(\sum_{S} f\left(a_{S}^{\prime}\right) a_{S}^{\prime \prime}\right)^{*} \\
& =\sum_{S}\left(f\left(a_{S}^{\prime}\right)\right)^{*}\left(a_{S}^{\prime \prime}\right)^{*} .
\end{aligned}
$$

On the other hand, the r.h.s. of Eq. (2.15) gives

$$
\begin{aligned}
a^{* * f *} f^{*} \kappa & =f^{*}\left(\kappa\left(a_{S}^{\prime *}\right)\right) a_{S}^{\prime \prime *} \\
& =\sum_{S}\left(f\left(\kappa\left(\kappa\left(a_{S}^{\prime *}\right)^{*}\right)\right)\right)^{*} a_{S}^{\prime \prime *} \\
& =\sum_{S}\left(f\left(a_{S}^{\prime}\right)\right)^{*} a_{S}^{\prime \prime *},
\end{aligned}
$$

where

$$
\Delta(a)=\sum_{S} a_{S}^{\prime} \otimes a_{S}^{\prime \prime}
$$

q.e.d.

With the help of Proposition 1, it is straightforward to show that Eqs. (2.10) and (2.11) are the $*$-conjugation of each other.

The definition of the bicovariant bimodule over $\mathscr{A}$ is completed by giving the functionals $f_{ \pm j}^{i}$. We give them as the value of functional $f_{ \pm j}^{i}$ when acting on the generators of $\mathscr{A}$, i.e., $M_{l}^{k}$ and $\bar{M}_{l}^{k}$.

Theorem 1. The complete definition of the functionals $f_{ \pm j}^{i}$ and $\bar{f}_{ \pm j}^{i}$ are:

$$
\begin{aligned}
f_{j}^{i}(\mathbf{I}) & =\delta_{j}^{i}, \\
\bar{f}_{j}^{l}(\mathbf{I}) & =\delta_{j}^{i}, \\
f_{ \pm j}^{i}\left(M_{l}^{k}\right) & =\hat{\mathbf{R}}_{l j}^{ \pm k}, \\
f_{ \pm j}^{i}\left(\bar{M}_{[l]}^{[k]}\right) & =\hat{\mathbf{R}}_{[l] j}^{i[k]}, \\
\bar{f}_{ \pm[j]}^{[i]}\left(M_{l}^{k}\right) & =\hat{\mathbf{R}}_{l[j]}^{-[i] k}, \\
\bar{f}_{ \pm[j]}^{[i]}\left(\bar{M}_{[l]}^{[k]}\right) & =\hat{\mathbf{R}}_{[l][j]}^{\mp[i][k]},
\end{aligned}
$$

where $\hat{\mathbf{R}}^{+}=\hat{\mathbf{R}}$ and $\hat{\mathbf{R}}^{-}=\hat{\mathbf{R}}^{-1}$ (see Appendix).

Proof. Equations (2.19) and (2.20) hold due to the fact that the unit element of the algebra commutes with any element of the bimodule.

The other equations are derived by requiring bicovariance as in the case [CSWW]. The derivation is performed in two steps. First we will prove Eqs. (2.21) and (2.22). Then, Eqs. (2.24) and (2.23) are derived by taking the $*$-conjugation of Eqs. (2.21) and (2.22), respectively.

We start the derivation of Eq. (2.22) with the relation of left and right multiplication of the $\bar{M}_{j}^{i}$ on the basis $\eta_{ \pm}^{i}$

$$
\eta_{ \pm}^{i} \bar{M}_{[l]}^{[k]}=\left(\bar{M}_{[l]}^{[k]} * f_{ \pm j}^{i}\right) \eta_{ \pm}^{j}=f_{ \pm j}^{i}\left(\bar{M}_{[\mathrm{s}]}^{[k]}\right) \bar{M}_{[l]}^{[s]} \eta_{ \pm}^{j}
$$


where we have used the definition of the convolution product. To fix the value of $f_{ \pm j}^{i}\left(\bar{M}_{l}^{k}\right)$ we examine the covariance of the above equation, i.e., we apply the left coaction on this equation and calculate it in two ways:

$$
\begin{aligned}
\Delta_{L}\left(\eta_{ \pm}^{i} \bar{M}_{[l]}^{[k]}\right) & =M_{i^{\prime}}^{i} \bar{M}_{\left[k^{\prime}\right]}^{[k]} \otimes \eta_{ \pm}^{i^{\prime}} \bar{M}_{[l]}^{\left[k^{\prime}\right]} \\
& =M_{i^{\prime}}^{i} \bar{M}_{\left[k^{\prime}\right]}^{[k]} \otimes f_{ \pm s}^{i^{\prime}}\left(\bar{M}_{[t]}^{\left[k^{\prime}\right]}\right) \bar{M}_{[l]}^{[t]} \eta_{ \pm}^{s},
\end{aligned}
$$

and applying the coaction on the r.h.s. of Eq. (2.25) we get

$$
\Delta_{L}\left(\eta_{ \pm}^{i} \bar{M}_{[l]}^{[k]}\right)=f_{ \pm i^{\prime}}^{i}\left(\bar{M}_{\left[k^{\prime}\right]}^{[k]}\right) \bar{M}_{[t]}^{\left[k^{\prime}\right]} M_{s}^{i^{\prime}} \otimes \bar{M}_{[l]}^{[t]} \eta_{ \pm}^{s} \text {. }
$$

Bicovariance means that both derivations must be equivalent and therefore we get the condition

$$
f_{ \pm i^{\prime}}^{i}\left(\bar{M}_{\left[j^{\prime}\right]}^{[j]}\right) \bar{M}_{[k]}^{\left[j^{\prime}\right]} M_{l}^{i^{\prime}}=M_{i^{\prime}}^{i} \bar{M}_{\left[j^{\prime}\right]}^{[j]} f_{ \pm l}^{i^{\prime}}\left(\bar{M}_{[k]}^{\left[j^{\prime}\right]}\right) .
$$

Thus, from Eq. (1.10) we conclude that

$$
f_{ \pm j}^{i}\left(\bar{M}_{[l]}^{[k]}\right)=\alpha \hat{\mathbf{R}}_{[l] j}^{i[k]},
$$

or equivalently

$$
f_{ \pm j}^{i}\left(\bar{M}_{l}^{k}\right)=\alpha \hat{\mathbf{R}}_{j l}^{-k i}
$$

where $\alpha$ is a nonzero constant.

The constant $\alpha$ is defined by the requirement that the $q$-determinant commutes with the basis $\eta_{ \pm}^{i}$ which implies

$$
f_{ \pm j}^{i}\left(\left(\operatorname{det}_{q} M\right)^{*}\right)=\delta_{j}^{i} .
$$

Using the definition of the $q$-determinant we get

$$
\begin{aligned}
f_{ \pm j}^{i}\left(\left(\operatorname{det}_{q} M\right)^{*}\right) & =\frac{(-1)^{N-1}}{\llbracket N \rrbracket} \varepsilon_{k_{1}} \ldots k_{N} f_{ \pm j}^{i}\left(\bar{M}_{l_{N}}^{k_{N}} \ldots \bar{M}_{l_{1}}^{k_{1}}\right) \varepsilon^{l_{1} \ldots l_{N}} \\
& =\frac{(-1)^{N-1}}{\llbracket N \rrbracket} \varepsilon_{k_{1}} \ldots k_{N} f_{ \pm i_{1}}^{i}\left(\bar{M}_{l_{N}}^{k_{N}}\right) f_{ \pm i_{2}}^{i_{1}}\left(\bar{M}_{l_{N-1}}^{k_{N-1}}\right) \ldots f_{ \pm j}^{i_{N-1}}\left(\bar{M}_{l_{1}}^{k_{1}}\right) \varepsilon^{l_{1} \ldots l_{N}} .
\end{aligned}
$$

Substituting Eq. (2.29') into (2.31) yields

$$
f_{ \pm j}^{i}\left(\left(\operatorname{det}_{q} M\right)^{*}\right)=\alpha^{N} \delta_{j}^{i} .
$$

Therefore the constant $\alpha$ satisfies

$$
\alpha^{N}=1 .
$$

It means the constant $\alpha$ is the $N^{\text {th }}$ root of unity. However in the limit $q \rightarrow 1$ the commutative case has to be reproduced. Therefore it follows that $\alpha=1$ and the result is Eq. (2.22).

The proof of Eq. (2.21) follows analogously to the one of the quantum group $\operatorname{Fun}_{q}(S U(N))$. We find two cases: the relation between the right and left multiplication is given either by $\hat{\mathbf{R}}$ or by $\hat{\mathbf{R}}^{-1}$. We distinguish these two cases by a suffix \pm of functional $f$, respectively. Note that we must also require the consistency of Eq. (1.6) with the bimodule structure. See ref. [CSWW]. 
To complete the proof of the theorem, we have to show that these definitions of the functionals are consistent with the other quantum group relations (1.7) and (1.10). For this we have to show that the equations

$$
\hat{\mathbf{R}}_{i j}^{i^{\prime} j^{\prime}}\left(\bar{M}_{j^{\prime}}^{l}, \bar{M}_{i^{\prime}}^{k} * f_{ \pm t}^{s}\right)=\left(\bar{M}_{j}^{j^{\prime}} \bar{M}_{i}^{i^{\prime}} * f_{ \pm t}^{s}\right) \hat{\mathbf{R}}_{i^{\prime} j^{\prime}}^{k l},
$$

and

$$
\left(\bar{M}_{i^{\prime}}^{i} M_{k}^{j^{\prime}} * f_{ \pm t}^{s}\right) \hat{\mathbf{R}}_{j^{\prime} l}^{-1 i^{\prime} j}=\hat{\mathbf{R}}_{k i^{\prime}}^{-1 i j^{\prime}}\left(M_{j^{\prime}}^{j} \bar{M}_{l}^{i^{\prime}} * f_{ \pm t}^{s}\right)
$$

hold also when applying the definitions of the functional (2.21) and (2.22) first. By using the property of the convolution product, the expression inside the bracket in Eqs. (2.34) and (2.35) can be rewritten using $\hat{\mathbf{R}}$ matrices. Then both relations above hold simply due to the braid relation (1.5) of the $\hat{\mathbf{R}}$ matrix.

To prove Eq. (2.24) we use the definition of the $*$-conjugation of the functional given in Eq. (2.14). Then Eq. (2.24) is rewritten as

$$
\begin{aligned}
\bar{f}_{ \pm j}^{i}\left(\bar{M}_{l}^{k}\right) & =f_{ \pm i}^{* j}\left(M_{k}^{l *}\right) \\
& =\left(f_{ \pm i}^{j}\left(\kappa\left(M_{k}^{l}\right)\right)\right)^{*} \\
& =\varepsilon^{l l_{1} \ldots l_{N-1}} f_{ \pm i}^{j}\left(M_{l_{1}}^{k_{1}} \ldots M_{l_{N-1}}^{k_{N-1}}\right)^{*} \varepsilon_{k_{1}, k_{N-1} k} .
\end{aligned}
$$

Using the $\hat{\mathbf{R}}$-matrix representation of the functional $f$ in Eq. (2.21) we see that the r.h.s. can be represented by the $\hat{\mathbf{R}}$ matrix as:

$$
\bar{f}_{ \pm j}^{i}\left(\bar{M}_{l}^{k}\right)=\hat{\mathbf{R}}_{l j}^{\mp i k} \text {. }
$$

Changing the suffix into the antisymmetrized index convention using Eqs. (1.17) and (1.18) and the definition of the $\hat{\mathbf{R}}$-matrix in Eq. (A.3), we get (2.24).

The proof of Eq. (2.23) can be performed analogously. q.e.d.

As already mentioned in the beginning we had as well the possibility of choosing $\hat{\mathbf{R}}$ instead of $\hat{\mathbf{R}}^{-1}$ in Eq. (1.10). Then, in Eqs. (2.22) and (2.23) the effect of taking the other choice of the relation between the $M$ and $\bar{M}$ in Eq. (1.10) would have been to convert $\hat{\mathbf{R}}$ into $\hat{\mathbf{R}}^{-1}$ and vice versa.

\section{The Algebra of the Bicovariant Bimodule}

Our aim is to construct the bicovariant differential calculus on the complex quantum group by generalizing Woronowicz's construction in ref. [Wor2]. Following the method developed in ref. [CSWW], we consider the differential calculus defined by the right invariant basis of the adjoint representation.

The construction of the adjoint representation in ref. [CSWW] is performed by taking the tensor product of two fundamental bimodules. We will proceed here in the same way.

The product of two bimodules $\eta_{1}$ and $\eta_{2}$ is understood to be defined over $\mathscr{A}$ :

$$
\eta_{1} \eta_{2} \equiv \eta_{1} \otimes_{\mathscr{A}} \eta_{2}
$$

We also extend the *-conjugation in a natural way as the antimultiplicative map:

$$
\left(\eta_{1} \eta_{2}\right)^{*}=\left(\eta_{2}\right)^{*}\left(\eta_{1}\right)^{*}
$$


In the present case, by using the analogy to the classical case, the adjoint representation can be obtained by using the $q$-antisymmetric tensor $\varepsilon_{i_{1}} \ldots i_{N}$ as

$$
\theta_{k}^{i} \equiv \eta^{i} \eta^{j_{1}} \ldots \eta^{j_{N-1}} \varepsilon_{j_{1} \ldots j_{N-1}} k
$$

or equivalently by using the upper suffix

$$
\theta^{i[k]} \equiv \eta^{i} \eta^{j_{1}} \ldots \eta^{j_{N-1}} \varepsilon_{j_{1} \ldots j_{N-1} l} \varepsilon^{l[k]},
$$

and their $*$-conjugation. It is easy to show that

$$
\Delta_{L}\left(\theta_{j}^{i}\right)=M_{i^{\prime}}^{i} \kappa\left(M_{j}^{j^{\prime}}\right) \otimes \theta_{j^{\prime}}^{i^{\prime}} .
$$

In Eq. (3.3) we did not write the suffix \pm for the basis since for any combination of the $\eta_{+}^{i}$ and $\eta_{-}^{i}$, the $\theta_{j}^{i}$ transforms as the adjoint representation. This additional structure did not appear in the case of $\mathrm{Fun}_{q}(S U(N))$, since the requirement of the unitarity fixed the choice of the basis. In the present case, the different choice of the combinations of $\eta_{ \pm}$in Eq. (3.3), for example $\eta_{+} \eta_{+} \eta_{-} \ldots \eta_{-}$, $\eta_{+} \eta_{-} \eta_{+} \ldots \eta_{-}$and etc., can either lead to different types of differential calculi or to such ones which are isomorphic to each other. This is the reason why in this section we examine the detailed structure of the product of bimodules and the tensor representations constructed by the fundamental bimodules $\eta_{ \pm}$and $\bar{\eta}_{ \pm}$.

First we consider the product of the same basis, i.e., the product of $\eta_{+}$with $\eta_{+}$ and $\eta_{-}$with $\eta_{-}$and their conjugate.

The product of two equal bimodules is reducible as the usual (non-deformed) representation and by using the projection operators $\mathscr{P}_{S}$ and $\mathscr{P}_{A}$ in Eq. (1.8) it can be decomposed into the irreducible representations:

$$
\eta_{+}^{i} \eta_{+}^{j}=\mathscr{P}_{S_{k l}^{i j}} \eta_{+}^{k} \eta_{+}^{l} \oplus \mathscr{P}_{A_{k l}^{i j}} \eta_{+}^{k} \eta_{+}^{l}
$$

and the same for the product of $\eta_{-}^{i}$.

The former choice gives the $q$-symmetric product, whereas the latter one gives the $q$-antisymmetric product. In both cases, it is easy to see that the product of the bases becomes again a bimodule over $\mathscr{A}$ and the resulting bimodule also splits into two parts.

Note that one can consider the algebra imposing either the symmetric or the antisymmetric combination to be zero like in the case of the $q$-plane algebra [Manin]. However, since these subalgebras contain only a part of the whole representation by definition, we do not impose such conditions since we want to have all kinds of tensor representations in the algebra.

When considering the product of different types of bases like $\eta_{+}$with $\eta_{-}$, or $\eta_{+}$ with $\bar{\eta}_{+}$, we find that multiplying the projection operator onto such products is not compatible with the bimodule structure since the relation of the left-right multiplication involves all components. On the other hand we find that the products of the same number of basis elements $\eta_{+}$and $\eta_{-}$but with different ordering generates equivalent bimodules with the right invariant bases given by these products. This situation can be represented compactly as commutation relations among the fundamental bimodules. Naturally the commutation relations must be bicovariant and must give consistency with the relation between the right and left multiplication given in the previous section.

For convenience we first list the resulting relations among the right invariant basis $\eta_{ \pm}^{i}$ and $\bar{\eta}_{ \pm i}$ : 
Theorem 2. Requiring the bicovariance, the possible commutation relations among the right invariant bases which coincide with the (anti-)commuting case in the limit $q \rightarrow 1$ are

$$
\begin{aligned}
\eta_{+}^{i} \eta_{-}^{j} & =\beta \hat{\mathbf{R}}_{k l}^{i j} \eta_{-}^{k} \eta_{+}^{l}, \\
\eta^{i} \bar{\eta}_{j} & =\gamma \hat{\mathbf{R}}_{l j}^{-1 k i} \bar{\eta}_{k} \eta^{l},
\end{aligned}
$$

where (3.8) holds for any combination of the suffix.$\pm \beta$ and $\gamma$ are nonzero constants which go to \pm 1 in the limit $q \rightarrow 1$.

The other relations among $\bar{\eta}_{ \pm i}$ are given by taking the *-conjugation of Eq. (3.7).

Proof. We consider the product of two bases belonging to different functionals, i.e. the product of $\eta_{+}^{l}$ and $\eta_{-}^{j}$. As in the case of two different $q$-planes as considered in ref. [CSSW], we can fix the commutation relations of these two different right invariant bases as follows. Take the ansatz

$$
\eta_{+}^{i} \eta_{-}^{j}=A_{k l}^{i j} \eta_{-}^{k} \eta_{+}^{l},
$$

where $A_{k l}^{i j} \in \mathbf{C}$. To impose the covariance we apply the coaction on both sides of Eq. (3.9). Then the 1.h.s. gives

$$
\Delta_{L}\left(\eta_{+}^{i} \eta_{-}^{j}\right)=M_{i^{\prime}}^{i} M_{j^{\prime}}^{j} \otimes \eta_{+}^{l^{\prime}} \eta_{-}^{j^{\prime}}=M_{i^{\prime}}^{i} M_{j^{\prime}}^{j} A_{k l}^{i^{\prime} j^{\prime}} \otimes \eta_{-}^{k} \eta_{+}^{l} .
$$

The r.h.s. gives

$$
\Delta_{L}\left(\eta_{+}^{i} \eta_{-}^{j}\right)=A_{k^{\prime} l^{\prime}}^{i j} M_{k}^{k^{\prime}} M_{l}^{l^{\prime}} \otimes \eta_{-}^{k} \eta_{+}^{l} .
$$

Comparing both equations, the condition of the covariance for $A_{k l}^{i j}$ is

$$
A_{k^{\prime} l^{\prime}}^{i j} M_{k}^{k^{\prime}} M_{l}^{l^{\prime}}=M_{i^{\prime}}^{i} M_{j^{\prime}}^{j} A_{k l}^{i^{\prime} j^{\prime}} .
$$

Then we get the solution: $A_{k l}^{i j}=\beta \hat{\mathbf{R}}_{k l}^{i j}+\beta^{\prime} \hat{\mathbf{R}}_{k l}^{-1 i j}$ with constants $\beta$ and $\beta^{\prime}$. Thus, as a possible commutation relation of $\eta_{+}^{i}$ and $\eta^{j}$ - we get

$$
\eta_{+}^{i} \eta_{-}^{j}=\left(\beta \hat{\mathbf{R}}_{k l}^{+i l}+\beta^{\prime} \hat{\mathbf{R}}_{k l}^{-i j}\right) \eta_{-}^{k} \eta_{+}^{l} .
$$

This is the result analogous to the case of the differential calculus on the $q$-Euclidean space [CSW]. There, the existence of the singlet component forces us to choose either $\hat{\mathbf{R}}$ or $\hat{\mathbf{R}}^{-1}$ in the commutation relation, and the choice of $\hat{\mathbf{R}}$ or $\hat{\mathbf{R}}^{-1}$ was simply a matter of convention. However, when we consider the bimodule structure of the $S L_{q}(N, C)$ we get a further condition which fixes the $\hat{\mathbf{R}}$-matrix of the above relation (3.13).

We require now that the product of two bases $\eta_{+}^{i} \eta^{j}$ - satisfying Eq. (3.13) is consistent with the basic commutation relations of the two fundamental bimodules, respectively. For this we examine the relations between left and right multiplication of $\mathscr{A}$ on this product of the bases.

For $a \in \mathscr{A}$

$$
\begin{aligned}
& \eta_{+}^{i} \eta_{-}^{j} a=\left(a * f_{-j^{\prime}}^{j} * f_{+i^{\prime}}^{i}\right) \eta_{+}^{i^{\prime}} \eta_{-}^{j^{\prime}}=\left(a * f_{-j^{\prime}}^{i} * f_{+i^{\prime}}^{i} A_{k l}^{i^{\prime} j^{\prime}}\right) \eta_{-}^{k} \eta_{+}^{l}, \\
& \eta_{+}^{i} \eta_{-}^{j} a=A_{k l}^{i j} \eta_{-}^{k} \eta_{+}^{l} a=A_{k^{\prime} l^{\prime}}^{i j}\left(a * f_{+l}^{l^{\prime}} * f_{-k}^{k^{\prime}}\right) \eta_{-}^{k} \eta_{+}^{l} .
\end{aligned}
$$


Comparing these two equations we have the following relation

$$
\left(a * f_{-j^{\prime}}^{j} * f_{+i^{\prime}}^{i}\right) A_{k l}^{i^{\prime} j^{\prime}}=A_{k^{\prime} l^{\prime}}^{i j}\left(a * f_{+l}^{l^{\prime}} * f_{-k}^{k^{\prime}}\right) .
$$

Taking $a$ to be a generator $a=M_{t}^{s}$ and using the property of the convolution product

$$
f_{-j^{\prime}}^{j}\left(M_{s^{\prime}}^{s}\right) f_{+i^{\prime}}^{i}\left(M_{t}^{s^{\prime}}\right) A_{k l}^{i^{\prime} j^{\prime}}=A_{k^{\prime} l^{\prime}}^{i j} f_{+l}^{l^{\prime}}\left(M_{s^{\prime}}^{\mathrm{s}}\right) f_{-k}^{k^{\prime}}\left(M_{t}^{s^{\prime}}\right) \text {. }
$$

Using Eq. (2.21) we see that Eq. (3.17) holds if and only if $A_{k l}^{i j}=\beta \hat{\mathbf{R}}_{k l}^{i j}$. With this choice Eq. (3.16) holds also for the case $a=\bar{M}_{t}^{s}$. Thus we get Eq. (3.7).

The commutation relation between $\eta_{ \pm}^{i}$ and $\bar{\eta}_{ \pm j}$ can be derived similarly. We again start with the most general ansatz:

$$
\eta^{i} \bar{\eta}_{j}=A_{l j}^{k i} \bar{\eta}_{k} \eta^{l}
$$

Taking the coaction on both sides to examine the covariance condition, we get the equation for $A_{l j}^{k i}$. The coaction on the 1.h.s. of Eq. (3.18) yields

$$
\Delta_{L}\left(\eta^{i} \bar{\eta}_{j}\right)=M_{i^{\prime}}^{i} \bar{M}_{j}^{j^{\prime}} \otimes \eta^{i^{\prime}} \bar{\eta}_{j^{\prime}}=M_{i^{\prime}}^{i} \bar{M}_{j}^{j^{\prime}} \otimes A_{l j^{\prime}}^{k i^{\prime}} \bar{\eta}_{k} \eta^{l} .
$$

The coaction on the r.h.s. of Eq. (3.18) gives

$$
\Delta_{L}\left(\eta^{i} \bar{\eta}_{j}\right)=A_{l^{\prime} j}^{k^{\prime} i} \bar{M}_{k^{\prime}}^{k} M_{l}^{l^{\prime}} \otimes \bar{\eta}_{k} \eta^{l} .
$$

Comparing Eqs. (3.19) and (3.20) we get

$$
A_{l^{\prime} j}^{k^{\prime} i} \bar{M}_{k^{\prime}}^{k} M_{l}^{l^{\prime}}=M_{i^{\prime}}^{i} \bar{M}_{j}^{j^{\prime}} A_{l j^{\prime}}^{k i^{\prime}}
$$

Comparing this result with Eq. (1.10) we conclude that $A_{k l}^{i j}$ is proportional to $\hat{\mathbf{R}}_{k l}^{-1 i j}$ and this leads to Eq. (3.8).

To complete the proof, we also examine the consistency of the relations between left and right multiplication of $\mathscr{A}$ with (3.8). We repeat the computation analogous to Eqs. (3.14)-(3.16). As a consistency condition we get

$$
\left(a * \bar{f}_{j^{\prime}}^{j} * f_{i^{\prime}}^{i}\right) \hat{\mathbf{R}}_{k l}^{-1 i^{\prime} j^{\prime}}=\hat{\mathbf{R}}_{k^{\prime} l^{\prime}}^{-1 i j}\left(a * f_{l}^{l^{\prime}} * \bar{f}_{k}^{k^{\prime}}\right) .
$$

We can confirm this equation straightforwardly by taking $a=M_{t}^{s}$ and $a=\bar{M}_{t}^{s}$ using the similar manipulation as done from Eq. (3.16) to Eq. (3.17). q.e.d.

Now when we consider the product of the basis $\eta_{ \pm}$and $\bar{\eta}_{ \pm}$, using the commutation relations (3.7) and (3.8), we can reorder the product, since we cannot distinguish them as bicovariant bimodules. The product depends now on the number of bases of each type.

Left-right invariant bicovariant bimodule. The left-right invariant elements play a key role when we define the exterior derivative. They will also enable us to show some important properties of the dual algebra. According to the result concerning the algebra of the bicovariant bimodule discussed above, one can find $2 \mathrm{~N}+2$ independent elements which are left-right invariants denoted by $\mathbf{X}_{(l)}$ and their *-conjugation counterparts. Explicitly, they are

$$
\mathbf{X}_{(l)} \equiv \varepsilon_{i_{1} \ldots i_{N}} \eta_{+}^{i_{1}} \ldots \eta_{+}^{i_{l}} \eta_{-}^{i_{l+1}} \ldots \eta_{-}^{i_{N}}
$$

where the suffix $l=0, \ldots, N$ counts the number of the bases with suffix + . 
Using the definition of the fundamental bimodule we can calculate the relation of the right and left multiplication of the element $a \in \mathscr{A}$ on $\mathbf{X}_{l}$ :

$\mathbf{X}_{(l)} a=\varepsilon_{l_{1} \ldots l_{N}}\left(a * f_{-j_{N}}^{l_{N}} * \ldots * f_{-j_{l+1}}^{i_{l+1}} * f_{+J_{l}}^{l_{l}} * \ldots * f_{+j_{1}}^{i_{1}}\right) \eta_{+}^{j_{1}} \ldots \eta_{+}^{j_{l}} \eta_{-}^{j_{l+1}} \ldots \eta_{-}^{j_{N}}$.

From the definition of the functional $f_{ \pm}$, we find the following result.

Proposition 2. The left-right invariant bases $\mathbf{X}_{(l)}$ commute with $\bar{M}_{j}^{i}$, i.e., for any $l=0 \ldots N$ :

$$
\mathbf{X}_{(l)} \bar{M}_{j}^{i}=\bar{M}_{j}^{i} \mathbf{X}_{(l)}
$$

Furthermore the elements $\mathbf{X}_{(0)}$ and $\mathbf{X}_{(N)}$ commute with any element of the algebra: $\forall a \in \mathscr{A}$ and $l=0$ or $l=N$

$$
\mathbf{X}_{(l)} a=a \mathbf{X}_{(l)} .
$$

Proof. Using Eq. (3.24) we get

$$
\begin{aligned}
\mathbf{X}_{(l)} \bar{M}_{m}^{k}= & \varepsilon_{i_{1} \ldots i_{N}} f_{-j_{N}}^{i_{N}}\left(\bar{M}_{m}^{k_{1}}\right) \ldots f_{-j_{l+1}}^{i_{l+1}}\left(\bar{M}_{k_{l-1}}^{k_{l}}\right) f_{+j_{l}}^{l_{l}}\left(\bar{M}_{k_{l}}^{k_{l+1}}\right) \\
& \ldots f_{+j_{1}}^{l_{1}}\left(\bar{M}_{k_{N-1}}^{k_{N}}\right) \bar{M}_{k_{N}}^{k} \eta_{+}^{j_{1}} \ldots \eta_{+}^{j_{l}} \eta_{-}^{j_{l+1}} \ldots \eta_{-}^{j_{N}} .
\end{aligned}
$$

From the definition of the $f_{ \pm}$in terms of the $\hat{\mathbf{R}}$ matrix given in Eq. (2.22) we have

$$
\varepsilon_{i_{1}} \quad \ldots i_{N} f_{-j_{N}}^{l_{N}}\left(\bar{M}_{m}^{k_{1}}\right) \ldots f_{-j_{l+1}}^{i_{l_{1}}}\left(\bar{M}_{k_{l-1}}^{k_{l}}\right) f_{+j_{l}}^{i_{l}}\left(\bar{M}_{k_{l}}^{k_{l+1}}\right) \ldots f_{+j_{1}}^{i_{1}}\left(\bar{M}_{k_{N-1}}^{k_{N}}\right)=\varepsilon_{j_{1}} \quad . j_{N} \delta_{m}^{k_{N}} .
$$

Thus in the r.h.s. of Eq. (3.27), the product of $N$ bases $\eta_{ \pm}^{j}$ becomes again $\mathbf{X}_{(l)}$ and we get Eq. (3.25).

The above result is due to the fact that $f_{+}(\bar{M})$ is independent of the suffix \pm and all is given only by $\hat{\mathbf{R}}^{+}$as in (2.22). On the other hand $f_{ \pm}(M)$ is given by either $\hat{\mathbf{R}}^{+}$or $\hat{\mathbf{R}}^{-}$depending on the suffix as (2.21). Therefore, $M_{j}^{i}$ does not commute with $\mathbf{X}_{(l)}$ for general $l$, except for $\mathbf{X}_{(0)}$ and $\mathbf{X}_{(N)}$ which consist of bases $\eta$ with the same suffix. Therefore the similar mechanism like (3.28) works again and we get

$$
\begin{aligned}
& \mathbf{X}_{(0)} M_{j}^{i}=M_{j}^{i} \mathbf{X}_{(0)}, \\
& \mathbf{X}_{(N)} M_{j}^{i}=M_{j}^{i} \mathbf{X}_{(N)} .
\end{aligned}
$$

This means that $\mathbf{X}_{(0)}$ and $\mathbf{X}_{(N)}$ commute with all generators of algebra $\mathscr{A}$. q.e.d.

Among the left-right invariant elements in the context of the differential calculus there are 4 special elements: $\mathbf{X}_{(1)}$ and $\mathbf{X}_{(N-1)}$ and their $*$-conjugates.

Since the properties of these singlet elements are important in order to understand the differential calculus and to discuss the relation to the $\operatorname{Fun}_{q}(S U(N))$ in the following section, let us examine them in detail.

$\mathbf{X}_{(1)}$ and $\mathbf{X}_{(N-1)}$ can also be represented as

$$
\mathbf{X}_{(1)}=\varepsilon_{i[j]} \eta_{+}^{i} \eta_{-}^{[j]},
$$

and

$$
\mathbf{X}_{(N-1)}=\varepsilon_{[i] j} \eta_{+}^{[i]} \eta_{-}^{j}
$$


where

$$
\eta_{ \pm}^{[i]} \equiv \eta_{ \pm}^{l_{1}} \ldots \eta_{ \pm}^{l_{N-1}} \varepsilon_{l_{1} \ldots l_{N-1}} \varepsilon^{j[i]}
$$

In the $\operatorname{Fun}_{q}(S U(N))$, these elements have been related to the conjugate representation [CSWW]:

$$
\eta_{ \pm}^{[i]} \sim \bar{\eta}_{\mp}^{[i]}=\bar{\eta}_{\mp j} \varepsilon^{j[i]} .
$$

Therefore, when considering the $\operatorname{Fun}_{q}(S U(N))$ the $\mathbf{X}_{(1)}$ is equivalent to the singlet element $\mathbf{X}$ defined in ref. [CSWW]. However in the $\operatorname{Fun}_{q}(\operatorname{SL}(N, C))$ case considered here, such an equivalence does not exist and we have to consider all bimodules constructed out of tensor products of the fundamental bimodules as independent objects.

The functional which gives the relation between the left and right multiplication on $\eta_{ \pm}^{[i]}$, which we call $F_{ \pm[j]}^{[i]}$ defined as

$$
\eta_{ \pm}^{[i]} a=\left(a * F_{ \pm[j]}^{[i]}\right) \eta_{ \pm}^{[j]}
$$

can be represented by the functional $f_{ \pm j}^{i}$ :

$$
F_{ \pm[j]}^{[i]}=\varepsilon_{[j] k} \kappa^{-1}\left(f_{ \pm l}^{k}\right) \varepsilon^{l[i]} .
$$

Using (2.21) and (2.22) we can derive the value of the functionals $F_{ \pm}$for the generators:

$$
\begin{aligned}
F_{ \pm[j]}^{[i]}\left(M_{l}^{k}\right) & =\hat{\mathbf{R}}_{l[i]}^{ \pm[i] k}, \\
F_{ \pm[j]}^{[i]}\left(\bar{M}_{[l]}^{[k]}\right) & =\hat{\mathbf{R}}_{[l][j]}^{[i][k]} .
\end{aligned}
$$

Here the functionals $F_{ \pm}$are again expressed in terms of the $R$-matrix. Comparing Eqs. (3.37) and (3.38) with Eqs. (2.23) and (2.24), we find that $F_{-[j]}^{[i]}$ has the same value as $\bar{f}_{-[j]}^{[i]}$ for $M_{j}^{i}$ and $\bar{M}_{j}^{i}$. It means that they are the same functional. This is already an indication for the fact that the number of functionals does not increase in the same manner as the number of independent bimodules does when considering the tensor product of the fundamental bimodules. We come back to this point in the next section.

\section{Dual Algebra and Relation to $\mathrm{Fun}_{q}(\mathrm{SU}(\mathrm{N}))$}

As in the case of the compact quantum groups discussed in ref. [CSWW], the algebra generated by the functionals $f_{ \pm j}^{i}$ and $\bar{f}_{ \pm j}^{i}$ can be interpreted as the Hopf algebra dual to the $\operatorname{Fun}_{q}(S L(N, C))$ with appropriate commutation relations which are the point of this section.

The coalgebra structure is induced straightforwardly through the definition of the functionals (2.10) and (2.11):

The coproduct, counit and antipode of the dual algebra are given due to the definition of the functional as in ref. [Wor2], by the matrix product.

$$
\begin{aligned}
& \Delta\left(f_{ \pm j}^{i}\right)=f_{ \pm k}^{i} \otimes f_{ \pm j}^{k}, \\
& \Delta\left(\bar{f}_{ \pm j}^{i}\right)=\bar{f}_{ \pm j}^{k} \otimes \bar{f}_{ \pm k}^{i}, \\
& \varepsilon\left(f_{ \pm j}^{i}\right)=\delta_{j}^{i} .
\end{aligned}
$$


We have also a natural definition of antipode:

$$
\kappa(f) \equiv f \circ \kappa
$$

The product of the dual algebra is defined by the convolution product [Wor2] and the unit is given by the counit of $\operatorname{Fun}_{q}(S L(N, C))$.

Therefore, the problem of defining the dual Hopf algebra is to find the consistent commutation relations among the functionals, i.e. the generators of the dual algebra. We have seen in the previous section that when we consider the product of two fundamental bimodules, the functional relating the left and right multiplication is given by the convolution product of the functionals $f_{ \pm j}^{i}$ and $\bar{f}_{ \pm j}^{i}$. This means that when we consider the commutation relations among the bimodules, we are implicitly considering also the commutation relations among the functionals. Actually, requiring that the algebra of these functionals must be consistent with the algebra of the bicovariant bimodule we can find the commutation relations among the functionals.

It can be shown that the following relations among the functionals follow from the algebra of the bimodule discussed in the previous section:

From the fact that the irreducible decomposition of the bimodule constructed by the product of the same basis splits correspondingly to the decomposition of (3.6), we find the relation

$$
\hat{\mathbf{R}}_{k^{\prime} l^{\prime}}^{i j} f_{ \pm l}^{l^{\prime}} * f_{ \pm k}^{k^{\prime}}=f_{ \pm j^{\prime}}^{j} * f_{ \pm i^{\prime}}^{i} \hat{\mathbf{R}}_{k l}^{i^{\prime} J^{\prime}}
$$

See also ref. [CSWW].

The other relations are given by

\section{Proposition 3.}

$$
\begin{aligned}
& \hat{\mathbf{R}}_{k^{\prime} l^{\prime}}^{i j} f_{ \pm l}^{l^{\prime}} * f_{-k}^{k^{\prime}}=f_{-j^{\prime}}^{j} * f_{+i^{\prime}}^{i} \hat{\mathbf{R}}_{k l}^{i^{\prime} j^{\prime}}, \\
& \hat{\mathbf{R}}_{l j^{\prime}}^{-1 i^{\prime}} \bar{f}_{j}^{j^{\prime}} * f_{i^{\prime}}^{i}=f_{l}^{l^{\prime}} * \bar{f}_{k^{\prime}}^{k} \hat{\mathbf{R}}_{l^{\prime} j}^{-1 k^{\prime} i}
\end{aligned}
$$

where Eq. (4.7) holds for all combinations of the suffix \pm .

Proof. The consistency of the relation (4.6) and (4.7) with the algebra of the bicovariant bimodule can be proven in a similar way. We show here Eq. (4.7).

For any element $a \in \mathscr{A}$, we can calculate the relation between the left and right multiplication of the bimodule as

$$
\eta^{i} \bar{\eta}_{j} a=\left(a * \bar{f}_{j}^{i^{\prime}} * f_{i^{\prime}}^{i}\right) \eta^{i^{\prime}} \bar{\eta}_{j^{\prime}}=\gamma\left(a * \bar{f}_{j}^{j^{\prime}} * f_{i^{\prime}}^{i}\right) \hat{\mathbf{R}}_{l j^{\prime}}^{-1 k i^{\prime}} \bar{\eta}_{k} \eta^{l}
$$

On the other hand, we can also calculate it in the different order,

$$
\eta^{i} \bar{\eta}_{j} a=\gamma \hat{\mathbf{R}}_{l j}^{-1 k i} \bar{\eta}_{k} \eta^{l} a=\gamma \hat{\mathbf{R}}_{l^{\prime} j}^{-1 k^{\prime} i}\left(a * f_{l}^{l^{\prime}} * \bar{f}_{k^{\prime}}^{k}\right) \bar{\eta}_{k} \eta^{l} .
$$

The consistency requires that both calculations must give the same result and comparing Eqs. (4.8) and (4.9) we get Eq. (4.7).

q.e.d.

As we know from the investigations of the $\operatorname{Fun}_{q}(S U(N))$ there is a condition on the determinant of the functionals. We can find this condition from the fact that 
$X_{(0)}$ and $X_{(N)}$ are commuting with all elements of the algebra $\mathbf{A}$, as we have seen in the previous section. This means that we can impose

$$
\begin{aligned}
& \varepsilon_{i_{1} \ldots i_{N}} f_{ \pm k_{N}}^{i_{N}} * \ldots * f_{ \pm k_{1}}^{l_{1}}=\varepsilon_{k_{1} \ldots k_{N}}, \\
& \varepsilon^{i_{1} \ldots i_{N}} \bar{f}_{ \pm i_{1}}^{k_{1}} * * \ldots * \bar{f}_{ \pm h}^{k_{N}}=\varepsilon^{k_{1} \ldots k_{N}} .
\end{aligned}
$$

This requirement can be written symbolically as

$$
\operatorname{det}_{q} f_{ \pm}=1
$$

where $\operatorname{det}_{q} f_{ \pm}$is defined as a possible overall factor in the r.h.s. of (4.10) similar as in (1.13).

In the case of the $\operatorname{Fun}_{q}(S U(N))$ due to the unitarity condition we had a further identity between $f_{ \pm}$and $\overline{f_{ \pm}}$(Proposition 3 in ref. [CSWW].) In the complex quantum group case we cannot impose the same relations since there is no unitarity condition. However still we can find similar relations among the functionals.

From the previous section we know that comparing Eqs. (3.37) and (3.38) with Eqs. (2.23) and (2.24) we see that we can perform the following identifications:

$$
F_{-[j]}^{[i]}=\bar{f}_{-[i]}^{[i]} .
$$

Using the relation (3.36), this identification can be written as

$$
\bar{f}_{-j}^{i}=\kappa^{-1}\left(f_{-j}^{i}\right) \text {. }
$$

Now we are in the situation to conclude our result about the dual algebra. We defined the commutation relation among the generators of the dual algebra by requiring the consistency with the algebra of the bicovariant bimodules discussed in the previous section. We have shown that Eqs. (4.5)-(4.7), (4.12) and (4.14) (and their *-conjugations) are the ones which satisfy the consistency requirement. However, from this approach we cannot exclude the possibility that there may still be some additional relations among the functionals.

Nevertheless, we conclude here that the Hopf algebra generated by the functionals $f$ and $\bar{f}$ with the above relations are the dual algebra of the complex quantum group defined in Sect. 1. The reason is that the relations listed above are the ones which define the complex quantum group $\operatorname{Fun}_{q}^{\prime}(S L(N, C))$ as the twisted product of the compact quantum group $\operatorname{Fun}_{q}(S U(N))$ and its dual $\operatorname{Fun}_{q}(S U(N))_{\text {reg }}^{*}$ as follows:

Like in the case of $\operatorname{Fun}_{q}(S U(N))$ [CSWW], comparing the Hopf algebra structure with the results obtained in $[\mathrm{RTF}]$ the algebra of $f_{+}$and $\bar{f}_{+}$can be identified with the algebra dual to the $\operatorname{Fun}_{q}(S U(N))$ by identifying the functionals with $L_{+}$and $L_{+i}^{* j}=\kappa^{-1}\left(L_{-j}^{i}\right)$ in ref. [RTF]: The values of the functional $f_{+}$and $\bar{f}_{+}$on the generator $\bar{M}_{j}^{i}$ given in Eqs. (2.22) and (2.24) are consistent with the unitarity condition of $\operatorname{Fun}_{q}(S U(N))$ and thus we cannot distinguish the algebra generated by $f_{+}$and $\bar{f}_{+}$from the dual algebra of $\operatorname{Fun}_{q}(S U(N))$ (see also [DSWZ]). On the other hand the defining equations of the functionals $f_{-}$and $\bar{f}_{-}$in Eqs. (2.22) and (2.24) are not compatible with the unitarity condition. However, the algebra of the functionals $f_{-}$and $\bar{f}_{-}$can be identified with the $\operatorname{Fun}_{q}(S U(N))$ itself. To see this we identify $f_{-j}^{* i}$ with the quantum matrix $M_{j}^{i}$ of $\operatorname{Fun}_{q}(S U(N))$. Then for example Eq. (4.14) is equivalent to the unitarity condition of the quantum matrix. 
Therefore the structure of $\operatorname{Fun}_{q}^{\prime}(S L(N, C))$, the dual algebra of the quantum group $\operatorname{Fun}_{q}(S L(N, C))$ is found to be the twisted product

$$
\operatorname{Fun}_{q}^{\prime}(S L(N, C))=\operatorname{Fun}_{q}(S U(N)) \tilde{\otimes} \operatorname{Fun}_{q}^{\prime}(S U(N))_{\mathrm{reg}}^{*} .
$$

It means that $\operatorname{Fun}_{q}^{\prime}(\operatorname{SL}(N, C))$ is generated by the generators of the two subalgebras and thus

$$
\operatorname{Fun}_{q}^{\prime}(S L(N, C)) \supset \operatorname{Fun}_{q}(S U(N)) \text { and } \operatorname{Fun}_{q}^{\prime}(S L(N, C)) \supset \operatorname{Fun}_{q}^{\prime}(S U(N))_{\mathrm{reg}}^{*} .
$$

On the other hand, the algebra is not the direct product of the two since the two subalgebras do not commute.

\section{Bicovariant Differential Calculus on Complex Quantum Group}

Using the result of the previous sections, now we can construct the bicovariant differential calculus on the complex quantum group $\operatorname{Fun}_{q}(S L(N, C))$. For this purpose, we introduce the basis of the right invariant bicovariant bimodule. We require that the basis contains the adjoint representation in the limit $q \rightarrow 1$ and therefore its general form is given by $\theta_{j}^{i}$ in Eq. (3.3) and its $*$-conjugated basis. The choice of the $\eta_{+}$and $\eta_{-}$in Eq. (3.3) must be defined so that the projection to the antisymmetric product appearing in the definition of the $\theta_{j}^{i}$ is consistent with the bimodule structure. Therefore we have the following two choices as the right invariant basis $\theta_{j}^{i}$ for the differential calculus:

$$
\theta^{i[k]} \equiv \eta_{+}^{i} \eta_{-}^{j_{1}} \ldots \eta_{-1}^{j_{N-1}} \varepsilon_{j_{1}} \cdot j_{N-1} l \varepsilon^{l[k]}
$$

or

$$
\theta^{i[k]} \equiv \eta_{-}^{i} \eta_{+}^{j_{1}} \cdots \eta_{+}^{j_{N-1}} \varepsilon_{j_{1}} \quad j_{v-1} l \varepsilon^{l[k]} .
$$

It is clear that the differential calculus based on the basis (5.2) is obtained by exchanging the suffix + and - of the calculus based on (5.1). Therefore, we take (5.1) as the definition of the basis $\theta_{j}^{i}$.

Using the definition (3.33), we can write $\theta^{i[k]}$ in (5.1) as

$$
\theta^{i[j]} \equiv \eta_{+}^{i} \eta_{-}^{[j]}
$$

Correspondingly we also introduce the $*$-conjugation of the basis $\theta_{j}^{i}$. Recalling our convention in Eqs. (1.17) and (1.18) we denote them as

$$
\bar{\theta}^{i[j]} \equiv \varepsilon^{i[l]}\left(\theta^{k[l]}\right)^{*} \varepsilon^{k[j]}=\varepsilon^{i l_{1}} l^{l_{N-1}} \bar{\eta}-l_{N-1} \ldots \bar{\eta}-l_{1} \bar{\eta}+{ }_{k} \varepsilon^{k[j]} .
$$

We define the bicovariant bimodule $\Gamma^{1}$ as the one which is generated by the bases $\theta_{j}^{l}$ and $\bar{\theta}_{j}^{l}$. In this way, the number of the bases of $\Gamma^{1}$ is $2 N^{2}$ rather than $2 N^{2}-2$ which is the dimension of the adjoint representation, since $\Gamma^{1}$ contains also two singlets. Like we discussed in ref. [CSWW], we cannot project out these singlets covariantly. On the other hand, these singlets play an important role in the following construction of the differential calculus. 
The relation between the left and right multiplication can be derived straightforwardly using (5.3) as

$$
\theta^{i[j]} a=\left(a * F_{-\left[j^{\prime}\right]}^{[j]} * f_{+i^{\prime}}^{i}\right) \theta^{i^{\prime}\left[j^{\prime}\right]},
$$

where we have used Eq. (3.35).

For the definition of the differential calculus the reversed relation of (5.5) appears frequently, therefore we introduce the following functionals $\mathbf{A}$ and $\tilde{\mathbf{A}}$ :

$$
\begin{aligned}
& a \theta^{i[j]}=\theta^{k[l]}\left(a * \mathbf{A}_{k[l]}^{i[j]}\right), \\
& a \bar{\theta}^{i[j]}=\bar{\theta}^{k[l]}\left(a * \tilde{\mathbf{A}}_{k[l]}^{i[j]}\right),
\end{aligned}
$$

where the functionals $\mathbf{A}$ and $\tilde{\mathbf{A}}$ are given by

$$
\begin{aligned}
& \mathbf{A}_{k[l]}^{i[j]} \equiv\left(F_{-[l]}^{[j]} * f_{+k}^{i}\right) \circ \kappa=\left(\kappa\left(f_{+k}^{i}\right) * f_{-n}^{m}\right) \varepsilon^{n[j]} \varepsilon_{[l] m}, \\
& \left.\widetilde{\mathbf{A}}_{k[l]}^{i[j]} \equiv\left(\bar{f}_{-k}^{i}\right) * \kappa\left(\bar{f}_{+n}^{m}\right)\right) \varepsilon^{n[j]} \varepsilon_{[l] m} .
\end{aligned}
$$

To define the exterior derivative, we need the left-right invariant forms. As we discussed in Sect. 3, we can construct such elements with $N$ bases $\eta$ and define as $\mathbf{X}_{(l)}$ in Eq. (3.23). Reminding the choice of the basis (5.3) for the differential calculus, the singlet elements which we must consider here are $\mathbf{X}_{(1)}$ and its conjugate. In terms of $\theta$ they are given by:

$$
\begin{aligned}
& \mathbf{X}_{(1)}=\varepsilon_{i[j]} \theta^{i[j]}, \\
& \overline{\mathbf{X}}_{(1)}=(-1)^{N} \varepsilon_{[j] i} \bar{\theta}^{i[j]}=\left(\mathbf{X}_{(1)}\right)^{*} .
\end{aligned}
$$

It was shown in ref. [CSWW] that the exterior derivative which is bicovariant and satisfies the Leibniz rule can be given by the commutator of an algebra element with the singlet part of the tensor product of two bases of the fundamental representation. We find that this construction is applicable for the present case and thus we define the exterior derivatives as

Definition 1. The exterior derivative $\mathbf{d}$ is the map

$$
\text { d: } \mathscr{A} \rightarrow \Gamma^{1}
$$

and for an element $a \in \mathscr{A}$ it is defined by

$$
\mathbf{d} a \equiv D a+\bar{D} a,
$$

where

$$
\begin{aligned}
D a & \equiv \frac{1}{\mathscr{N}}\left[a, \mathbf{X}_{(1)}\right], \\
\bar{D} a & \equiv \frac{-1}{\mathscr{N}}\left[a, \overline{\mathbf{X}}_{(1)}\right],
\end{aligned}
$$

where the normalization constant $\mathscr{N}$ is real and proportional to $q-q^{-1}$ in the limit $q \rightarrow 1$ otherwise the commutator vanishes with this limit. (About this constant see also ref. [CSWW].) Note that $\bar{D} a=\left(D a^{*}\right)^{*}$. 
Since the exterior derivative is defined as a commutator, it satisfies automatically the Leibniz rule

$$
\mathbf{d}(a b)=(\mathbf{d} a) b+a(\mathbf{d} b)
$$

where $a, b \in \mathscr{A}$. Under the $*$-conjugation, it behaves as

$$
(\mathbf{d} a)^{*}=\mathbf{d} a^{*} .
$$

Higher differential forms. After fixing the basis of the right invariant 1-form, we define the higher differential forms. For this we define the wedge product of the basis $\theta_{j}^{i}$ and $\bar{\theta}_{j}^{i}$ and consider the bimodule $\Gamma^{p}\left(=\Gamma^{1} \wedge \ldots \wedge \Gamma^{1}, p\right.$ times $)$ with the basis of $p^{\text {th }}$ order $q$-antisymmetric product using this wedge product [Wor2]. From the analysis given in ref. [CSWW], knowing the commutation relation among the bases $\eta$, the $q$-antisymmetric product of $\theta$ can be defined by the combination of the symmetric and antisymmetric product of each $\eta$ appearing in the definition of $\theta$. Therefore, we define the wedge product as

Definition 2. The wedge product of two $\theta_{j}^{i}$ is defined as the q-antisymmetric tensor product over $\mathscr{A}$ :

$$
\theta_{k}^{i} \wedge \theta_{l}^{j} \equiv \theta_{k}^{i} \otimes_{\mathscr{A}} \theta_{l}^{j}
$$

with the condition of the vanishing of the q-symmetric combination:

$$
\begin{gathered}
\left(\mathscr{P}_{A}, \tilde{\mathscr{P}}_{A}\right)_{k_{0}[k] l_{0}[l]}^{l_{0}[i] j_{0}[j]}\left(\theta^{k_{0}[k]} \wedge \theta^{l_{0}[l]}\right)=0, \\
\left(\mathscr{P}_{S}, \tilde{\mathscr{P}}_{S}\right)_{k_{0}[k] l_{0}[l]}^{i_{0}[i] j_{0}[j]}\left(\theta^{k_{0}[k]} \wedge \theta^{l_{0}[l]}\right)=0,
\end{gathered}
$$

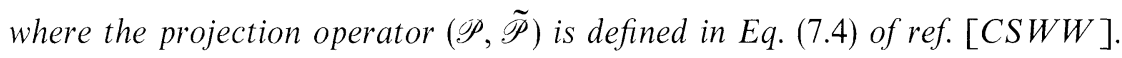

The wedge product among the $\bar{\theta}_{j}^{i}$ can be obtained by taking the $*$-conjugation of Eqs. (5.19) and (5.20) where the $*$-conjugation of a $p$-form $\rho$ and a $p^{\prime}$-form $\rho^{\prime}$ is defined by

$$
\left(\rho \wedge \rho^{\prime}\right)^{*}=(-1)^{p p^{\prime}} \rho^{*} \wedge \rho^{*} .
$$

In order to define the wedge product of $\theta_{j}^{i}$ and $\bar{\theta}_{j}^{i}$ we cannot use the above method since we do not have the definition of the $q$-(anti)symmetric product of $\eta$ and $\bar{\eta}$. Instead we have the consistent commutation relation among them as given in Eqs. (3.7) and (3.8). Using these we can find the covariant commutation relation between $\theta_{j}^{i}$ and $\bar{\theta}_{j}^{i}$ :

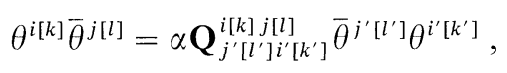

where $\alpha$ is a constant and $\mathbf{Q}$ is defined in terms of the $\hat{\mathbf{R}}$-matrix as

$$
\mathbf{Q}_{j^{\prime}\left[l^{\prime}\right] i^{\prime}\left[k^{\prime}\right]}^{i[j]\left[\left[j^{\prime}\right.\right.}=\varepsilon_{[m] j^{\prime}} \hat{\mathbf{R}}_{s\left[m^{\prime}\right]}^{-1[m] i} \hat{\mathbf{R}}_{\left[k^{\prime \prime}\right]\left[j^{\prime \prime}\right]}^{-1\left[m^{\prime}\right][k]} \varepsilon^{j\left[j^{\prime \prime}\right]} \hat{\mathbf{R}}_{\left[l^{\prime}\right] i^{\prime}}^{s\left[l^{\prime \prime}\right]} \hat{\mathbf{R}}_{\left[l^{\prime \prime}\right]\left[k^{\prime}\right]}^{\left[k^{\prime \prime}\right][l]} .
$$

It is clear that the wedge product which is bicovariant must satisfy the same commutation relation as (5.22) with an appropriate constant $\alpha$. It turns out that the constant $\alpha$ can be determined by requiring the nilpotency of the exterior derivative. We define the exterior derivative of the $p$-form as 
Definition 3. The exterior derivative is the map

$$
\text { d: } \Gamma^{p} \rightarrow \Gamma^{p+1}
$$

where $\mathbf{d}$ is defined by $\mathbf{d}=D+\bar{D}$ and for the $p$-form $\rho$ it acts as

$$
\begin{aligned}
& D \rho \equiv \frac{1}{\mathscr{N}}\left[\rho \wedge \mathbf{X}_{(1)}\right]_{ \pm}=\frac{1}{\mathscr{N}}\left(\rho \wedge \mathbf{X}_{(1)}-(-1)^{p} \mathbf{X} \wedge \rho\right), \\
& \bar{D} \rho \equiv \frac{-1}{\mathscr{N}}\left[\rho \wedge \overline{\mathbf{X}}_{(1)}\right]_{ \pm},
\end{aligned}
$$

where $[\cdot, \cdot]_{ \pm}$denotes the graded commutator with wedge product.

The above exterior derivative satisfies the Leibniz rule

$$
\mathbf{d}\left(\rho \wedge \rho^{\prime}\right)=(\mathbf{d} \rho) \wedge \rho^{\prime}+(-1)^{p} \rho \wedge\left(\mathbf{d} \rho^{\prime}\right),
$$

where $\rho \in \Gamma^{p}$ and $\rho^{\prime} \in \Gamma^{p^{\prime}}$. Under the $*$-conjugation, it behaves as

$$
(\mathbf{d} \rho)^{*}=\mathbf{d} \rho^{*} .
$$

With this definition, we can prove the following theorem.

Proposition 4. Defining the wedge product of the $\theta_{j}^{i}$ and $\bar{\theta}_{j}^{i}$ as

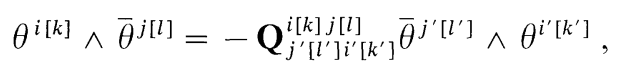

and taking Eqs. (5.19) and (5.20) for the wedge product of $\theta_{j}^{i}$ the exterior derivative $\mathbf{d}=D+\bar{D}$ satisfies

$$
\mathbf{d}^{2}=(D+\bar{D})^{2}=0
$$

Proof. It is sufficient to prove that

$$
\left(\mathbf{X}_{(1)}+\overline{\mathbf{X}}_{(1)}\right) \wedge\left(\mathbf{X}_{(1)}+\overline{\mathbf{X}}_{(1)}\right)=0 \text {. }
$$

Since Eqs. (13.2) and (13.3) are the same relation as the wedge product among the right invariant bases in the case of $\operatorname{Fun}_{q}(S U(N))$ in ref. [CSWW], we get

$$
\mathbf{X}_{(1)} \wedge \mathbf{X}_{(1)}=0 \text {, }
$$

and thus $D^{2}=0$. Taking the $*$-conjugation we also get

$$
\overline{\mathbf{X}}_{(1)} \wedge \overline{\mathbf{X}}_{(1)}=0
$$

which implies $\bar{D}^{2}=0$.

Therefore in order to prove Eq. (5.31) we have to prove that (5.29) implies

$$
\mathbf{X}_{(1)} \wedge \overline{\mathbf{X}}_{(1)}+\overline{\mathbf{X}}_{(1)} \wedge \mathbf{X}_{(1)}=0 \text {. }
$$

For this we need the following property of the matrix $\mathbf{Q}$ :

$$
\varepsilon_{i[k]} \varepsilon_{[l] j} \mathbf{Q}_{j^{\prime}\left[l^{\prime}\right] i^{\prime}\left[k^{\prime}\right]}^{i[k] j[l]}=\varepsilon_{i^{\prime}\left[k^{\prime}\right]} \varepsilon_{\left[l^{\prime}\right] j^{\prime}} .
$$

Using this relation and (5.29) we get (5.34). q.e.d.

Therefore Eqs. (5.19), (5.20) and (5.29) give the definition of the wedge product and the exterior derivative becomes nilpotent as (5.30). 
Note that in the present case the requirement of the nilpotency of the exterior derivative is essential. On the contrary, in the construction of the differential calculus on $\operatorname{Fun}_{q}(S U(N))$ given in ref. [CSWW], the nilpotency of the exterior derivative gives no new condition.

By the definition of the derivatives $D$ and $\bar{D}$, Eqs. (5.34) implies that

$$
D \bar{D}+\bar{D} D=0 \text {. }
$$

Furthermore we can prove that $\mathbf{X}_{(1)}\left(\overline{\mathbf{X}}_{(1)}\right)$ commutes with any of the $\bar{\theta}_{j}^{i}\left(\theta_{j}^{i}\right)$ and thus we get

$$
D \bar{\theta}_{j}^{i}=0 \quad \text { and } \quad \bar{D} \theta_{j}^{i}=0 .
$$

Using these results we can consider the Lie derivatives on $\operatorname{Fun}_{q}(S L(N, C))$ and examine their properties. For the complex quantum group, the right invariant vector fields $\chi_{i[j]}$ and $\tilde{\chi}_{i[j]}$ are introduced by

$$
\begin{aligned}
& D a=\theta^{k[l]}\left(a * \chi_{k[l]}\right), \\
& \bar{D} a=\bar{\theta}^{k[l]}\left(a * \tilde{\chi}_{k[l]}\right),
\end{aligned}
$$

where

$$
\begin{aligned}
& \chi_{k[l]} \equiv \varepsilon_{i[j]} \mathbf{A}_{k[l]}^{i[j]}-\varepsilon_{k[l]} \varepsilon, \\
& \tilde{\chi}_{k[l]} \equiv \varepsilon_{[j] i} \tilde{\mathbf{A}}_{k[l]}^{i[j]}-\varepsilon_{[l] k} \varepsilon .
\end{aligned}
$$

The Leibniz rule of the Lie derivative $(\cdot * \chi)$, i.e., coproduct of the functional $\chi$ is

$$
\begin{aligned}
& \Delta\left(\chi_{i[j]}\right)=\left(\chi_{i[j]} \otimes \varepsilon\right)+\left(\mathbf{A}_{i[j]}^{k[l]} \otimes \chi_{k[l]}\right), \\
& \Delta\left(\tilde{\chi}_{i[j]}\right)=\left(\tilde{\chi}_{i[j]} \otimes \varepsilon\right)+\left(\tilde{\mathbf{A}}_{i[j]}^{k[l]} \otimes \tilde{\chi}_{k[l]}\right) .
\end{aligned}
$$

These Lie derivatives satisfy the $q$-deformed commutation relations which can be derived by using the Maurer-Cartan equation. We briefly give the results here. In the present calculus the Maurer-Cartan equation is

$$
\begin{aligned}
& D \theta^{I}=\mathscr{F}_{J K}^{I} \theta^{J} \wedge \theta^{K}, \\
& D \bar{\theta}^{I}=0,
\end{aligned}
$$

and the $*$-conjugation of these equations. Here, the upper case suffix $I$ denotes the pair $i_{0}[i]$ with projection to the adjoint representation:

$$
V^{I} \equiv V^{i_{0}[i]}-\frac{(-1)^{N-1}}{\llbracket N \rrbracket} \varepsilon^{i_{0}[i]} \varepsilon_{j[k]} V^{j[k]} .
$$

The structure constant $\mathscr{F}_{J K}^{I}$ is given by

$$
\mathscr{F}_{J K}^{I}=\frac{\left(q-q^{-1}\right)(-1)^{N-1}}{\mathscr{N}\left(q^{N}-\frac{\left(q-q^{-1}\right)}{\llbracket N \rrbracket}\right)} F_{J K}^{I},
$$

where $F_{J K}^{I}$ is given in Eq. (7.16) of ref. [CSWW] replacing $\varepsilon_{\left[i^{\prime}\right] k_{0}}$ with the one in Eq. (1.12) in Sect. 1. 
Using the projection to the adjoint representation, we can decompose the derivative of $a \in \mathscr{A}$ as

$$
D a=\theta^{I}\left(a * \chi_{I}\right)+\frac{(-1)^{N-1}}{\llbracket N \rrbracket} \mathbf{X}\left(a * \chi_{0}\right),
$$

where $\chi_{0}=\chi_{i[j]} \varepsilon^{i[j]}$. We can prove that $\chi_{0}$ commutes with other functionals $\chi_{I}$ and $\tilde{\chi}_{I}$. Then we get

$$
D^{2} a=\theta^{J} \wedge \theta^{K}\left(a * \hat{\mathscr{F}}_{J K}^{I} * \chi_{I}\right)-\theta^{I} \wedge \theta^{J}\left(a * \chi_{I} * \chi_{J}\right)
$$

where the functional $\hat{\mathscr{F}}_{J K}^{I}$ is defined by

$$
\hat{\mathscr{F}}_{J K}^{I}=\mathscr{\mathscr { F }}_{J K}^{I}\left(\varepsilon-\frac{(-1)^{N-1}}{\llbracket N \rrbracket} \mathscr{N} \chi_{0}\right) .
$$

Therefore we obtain the commutation relation of the functional $\chi$ as

$$
\left[\chi_{J}, \chi_{K}\right]_{R}=\hat{\mathscr{F}}_{J K}^{I} * \chi_{I}
$$

where the commutator $[\cdot, \cdot]_{R}$ is defined as

$$
\left[\chi_{K}, \chi_{L}\right]_{R}=\left(\left(\mathscr{P}_{S}, \tilde{\mathscr{P}}_{A}\right)+\left(\mathscr{P}_{A}, \tilde{\mathscr{P}}_{S}\right)\right)_{K L}^{I J}\left(\chi_{I} * \chi_{J}\right) .
$$

From (5.45) we get the relation

$$
\bar{\chi}_{I} * \chi_{J}-\mathbf{Q}_{I J}^{K L} \chi_{K} * \bar{\chi}_{L}=0
$$

Relation to Quantum Space Algebra. In the rest of this section, we want to give a method to represent the above differential calculus on the quantum group by means of the algebra of the quantum space. Especially, here we write the action of the Lie derivative on the quantum space by the $q$-commutator of comodules. This provides the direct translation of the differential calculus on the quantum group as the infinitesimal transformation of the quantum space represented by the differential operators on this space. This will also be helpful to compare our result with the approach taken by [SWZ].

The quantum space algebra $\mathscr{M}$ is generated by the coordinate functions $z^{i}$ which give a left-comodule over $\mathscr{A}$ [Manin]:

$$
\Delta_{L}\left(z^{i}\right)=M_{j}^{i} \otimes z^{j}
$$

and satisfy the commutation relations

$$
\mathscr{P}_{A k l}^{i j} z^{k} z^{l}=0 .
$$

In order to connect the differential calculus on the quantum group with the quantum space algebra, first we define the product $*$ of an element of quantum space algebra $\mathscr{M}$ and a functional $f \in \mathscr{A}^{\prime}$ :

$$
*: \mathscr{M} \times \mathscr{A}^{\prime} \rightarrow \mathscr{M},
$$

which is the generalization of the convolution product. [Analogous formula is also used in ref. $[\operatorname{Pod} 2]$ in connection with the differential calculus on the quantum sphere.] 
Definition 4: $\forall x \in \mathscr{M}$ and $\forall f \in \mathscr{A}^{\prime}$

$$
(x * f) \equiv(f \otimes \mathrm{id}) \Delta_{L}(x) .
$$

By this we can define the action of the functional $f \in \mathscr{A}^{\prime}$ on the quantum space algebra $\mathscr{M}$. And using this definition we define the action of the exterior derivative $D$ and $\bar{D}$ on $\mathscr{M}$ which can be interpreted as the infinitesimal transformation with parameter $\theta^{i[j]}$ and $\bar{\theta}^{i[j]}$ respectively. We require that the derivative acts on the quantum space algebra covariantly, i.e., the coaction and derivative satisfy the relation

$$
\Delta_{L}(\mathbf{d} x) \equiv(\mathrm{id} \otimes \mathbf{d}) \Delta_{L}(x) .
$$

A natural definition which satisfies this requirement is:

Definition 5. We define the action of the derivative operator $\mathbf{d}=D+\bar{D}$ on an element $x \in \mathscr{M}$ by

$$
\begin{aligned}
& D x \equiv \theta^{i[j]}\left(x * \gamma_{i[j]}\right), \\
& \bar{D} x \equiv \bar{\theta}^{i[j]}\left(x * \tilde{\chi}_{i[j]}\right) .
\end{aligned}
$$

We also require that the Leibniz rule of $\mathbf{d}$ acting on $\mathscr{M}$ is

$$
\mathbf{d}(x y)=(\mathbf{d} x) y+x(\mathbf{d} y),
$$

for $x, y \in \mathscr{M}$.

Proposition 5. With Definition 5, the Leibniz rule (5.60) holds by setting the following bicovariant commutation relations:

$$
\begin{aligned}
& x \theta^{i[j]}=\theta^{k[l]}\left(x * \mathbf{A}_{k[l]}^{i[j]}\right), \\
& x \bar{\theta}^{i[j]}=\bar{\theta}^{k[l]}\left(x * \tilde{\mathbf{A}}_{k[l]}^{i[j]}\right),
\end{aligned}
$$

where the bicovariance is imposed by the left coaction on the product of $\forall x, y \in \mathscr{M}$ and $\omega=\theta^{i[j]}$ or $\bar{\theta}^{i[j]}$ being defined as

$$
\Delta_{L}(x \omega y)=\Delta_{\mathrm{L}}(x) \Delta_{L}(\omega) \Delta_{L}(y) .
$$

Proof. The 1.h.s. of Eq. (5.60) is given by $\mathbf{d}(x y)=D(x y)+\bar{D}(x y)$, where

$$
\begin{aligned}
D(x y) & =\theta^{i[j]}\left(\left(\chi_{i[j]} \otimes \mathrm{id}\right) \Delta_{L}(x y)\right) \\
& =\theta^{i[j]}\left(\left(\chi_{i[j]} \otimes \mathrm{id}\right) \Delta_{L}(x) \Delta_{L}(y)\right) \\
& =\theta^{i[j]}\left(\left(x * \chi_{i[j]}\right) y+\left(x * \mathbf{A}_{i[j]}^{k[l]}\right)\left(y * \chi_{k[l]}\right)\right),
\end{aligned}
$$

and correspondingly

$$
\bar{D}(x y)=\bar{\theta}^{i[j]}\left(\left(x * \tilde{\chi}_{i[j]}\right) y+\left(x * \tilde{\mathbf{A}}_{i[j]}^{k[l]}\right)\left(y * \tilde{\chi}_{k[l]}\right)\right) .
$$

To derive above expressions we have used the definition in Eqs. (5.58) and (5.59), Eq. (5.56) and the coproducts of $\chi$ and $\bar{\chi}$ given in Eqs. (5.42) and (5.43). 
On the other hand the r.h.s. of Eq. (5.60) is given by the summation of the following two terms:

$$
\begin{aligned}
& (D x) y+x(D y)=\theta^{i[j]}\left(x * \chi_{i[j]}\right) y+x \theta^{i[j]}\left(y * \chi_{i[j]}\right) \\
& (\bar{D} x) y+x(\bar{D} y)=\bar{\theta}^{i[j]}\left(x * \tilde{\chi}_{i[j]}\right) y+x \bar{\theta}^{i[j]}\left(y * \tilde{\chi}_{i[j]}\right) .
\end{aligned}
$$

Comparing Eqs. (5.66) and (5.67) with Eqs. (5.64) and (5.65) respectively and using the fact that they hold for any element $x, y$, we can conclude Proposition 5. The bicovariance of the relation is clear from the bicovariance of the relations (5.6) and (5.7).

q.e.d.

In the following we show that we can represent this operation with a commutator among the comodules. We consider a second rank tensor $\nabla_{i[j]}$ together with coordinate functions. The tensor $\nabla_{i[j]}$ does not commute with the elements of the quantum space algebra and the commutator gives the generalization of the infinitesimal transformation. Therefore, this tensor $\nabla_{i[j]}$ can be interpreted as a differential operator on the quantum space.

Definition 6. We introduce the tensor $\nabla_{i[j]}$ as an operator acting on $\mathscr{M}$ which satisfies the relation

$$
\begin{aligned}
& {\left[\nabla_{i[j]}, x\right]_{q} \equiv\left(x * \dot{\chi}_{i[j]}\right),} \\
& {\left[\tilde{\nabla}_{i[j]}, x\right]_{q} \equiv\left(x * \tilde{\chi}_{i[j]}\right),}
\end{aligned}
$$

where $x \in \mathscr{M}$ and $[\cdot, \cdot]_{q}$ is defined by

$$
\begin{aligned}
& {\left[\nabla_{i[j]}, x\right]_{q}=\nabla_{i[j]} x-\left(x * \mathbf{A}_{i[j]}^{k[l]}\right) \nabla_{k[l]},} \\
& {\left[\tilde{\nabla}_{i[j]}, x\right]_{q}=\tilde{\nabla}_{i[j]} x-\left(x * \tilde{\mathbf{A}}_{i[j]}^{k[l]}\right) \tilde{\nabla}_{k[l]} .}
\end{aligned}
$$

Before we prove the consistency of Definition 6 with the bicovariance, we explain how this definition encodes the action and the coproduct of the Lie derivative $\chi$ and $\bar{\gamma}$. Actually, the definition of the $q$-deformed commutator in the above definition is determined so that the Leibniz rule (5.60) is satisfied naturally in the new algebra. This is shown by direct computation of the action of the $\nabla_{i[j]}$ on the product of the elements $x, y \in \mathscr{M}$ :

$$
\begin{aligned}
{\left[\nabla_{i[j]}, x y\right]_{q} } & =\nabla_{i\lfloor j]} x y-\left(x y * A_{i[j]}^{k[l]}\right) \nabla_{k[l]} \\
& =\left(\left[\nabla_{i[j]}, x\right]_{q}+\left(x * A_{i[j]}^{s[t]}\right) \nabla_{s[t]}\right) y-\left(x * A_{i[j]}^{s[t]}\right)\left(y * A_{s[t]}^{k[l]}\right) \nabla_{k[l]} \\
& =\left[\nabla_{i[j]}, x\right]_{q} y+\left(x * \mathbf{A}_{i[j]}^{k[l]}\right)\left[\nabla_{k[l]}, y\right]_{q},
\end{aligned}
$$

where from the first line to the second we have used Eq. (5.70) and the relation $\Delta\left(A_{i[j]}^{k[l]}\right)=A_{i[j]}^{s[t]} \otimes A_{s[t]}^{k[l]}$. From the second line to the third we have used again Eq. (5.56) for $\nabla_{s[t]} y$.

The corresponding equation for $\tilde{\nabla}_{i[j]}$ is derived in the same way. The result is

$$
\left[\tilde{\nabla}_{i[j]}, x y\right]_{q}=\left[\tilde{\nabla}_{i[j]}, x\right]_{q} y+\left(x * \tilde{\mathbf{A}}_{i[j]}^{k[l]}\right)\left[\tilde{\nabla}_{k[l]}, y\right]_{q} .
$$


By virtue of definitions (5.68) and (5.69) the derivative in Eqs. (5.58) and (5.59) are now represented as

$$
\begin{aligned}
& D x \equiv \theta^{i[j]}\left[\nabla_{i[j]}, x\right]_{q}, \\
& \bar{D} x \equiv \bar{\theta}^{i[j]}\left[\tilde{\nabla_{i[j]}}, x\right]_{q} .
\end{aligned}
$$

Then Eqs. (5.72) and (5.73) imply that the relations which are given by substituting (5.68) and (5.69) into Eqs. (5.64) and (5.65), respectively, also hold. Hence, the Leibniz rule (5.60) is valid when we define the derivative as $\mathbf{d} x=D x+\bar{D} x$ with $D x$ and $\bar{D} x$ in (5.74) and (5.75).

To show that the algebra generated by $\nabla_{i[j]}$ and $\tilde{\nabla}_{i[j]}$ together with the coordinate functions $z^{i}$ and $\bar{z}_{i}$ is also covariant under the quantum group transformation, we have to show the consistency of Definition 6 with the bicovariance. This can be achieved by the following property of the tensors $\nabla_{i[j]}$ and $\tilde{\nabla}_{i[j]}$ as a left-comodule:

Proposition 6. If we define the coaction for $\nabla_{i[j]}$ and $\tilde{\nabla}_{i[j]}$ as

$$
\begin{aligned}
& \Delta_{L}\left(\nabla_{i[j]}\right)=\kappa^{-1}\left(M_{i}^{k} \kappa\left(M_{[j]}^{[l]}\right)\right) \otimes \nabla_{k[l]}, \\
& \Delta_{L}\left(\tilde{\nabla}_{i[j]}\right)=\kappa^{-1}\left(\kappa\left(\bar{M}_{i}^{k}\right) \bar{M}_{[j]}^{[l]}\right) \otimes \tilde{\nabla}_{k[l]},
\end{aligned}
$$

then the commutators with $x \in \mathscr{M}$ defined in Eqs. (5.70) and (5.71) satisfy

$$
\begin{aligned}
& \Delta_{L}\left(\left[\nabla_{i[j]}, x\right]_{q}\right)=\sum_{s}\left(\kappa^{-1}\left(M_{i}^{k} \kappa\left(M_{[j]}^{[l]}\right)\right) a_{s} \otimes\left[\nabla_{k[l]}, x_{s}\right]_{q}\right), \\
& \Delta_{L}\left(\left[\tilde{\nabla}_{i[j]}, x\right]_{q}\right)=\sum_{s}\left(\kappa^{-1}\left(\kappa\left(\bar{M}_{i}^{k}\right) \bar{M}_{[j]}^{[l]}\right) a_{s} \otimes\left[\tilde{\nabla}_{k[l]}, x_{s}\right]_{q}\right),
\end{aligned}
$$

where $a_{\mathrm{s}} \in \mathscr{A}$ and $x_{s} \in \mathscr{M}$ and $\Delta_{L}(x)=\sum_{s}\left(a_{s} \otimes x_{s}\right)$.

Proof. Since the coaction on the first term in Eq. (5.70) and in Eq. (5.71) has the same form as Eq. (5.78) and (5.79), respectively by definition, we have to prove that the second term in Eq. (5.70) and in Eq. (5.71) behaves in the same way under the coaction. For this we use the relation: $\forall a \in \mathscr{A}$

$$
\begin{aligned}
& \left(a * \mathbf{A}_{i[j]}^{k[l]}\right) \kappa^{-1}\left(M_{k}^{m} \kappa\left(M_{[l]}^{[n]}\right)\right)=\kappa^{-1}\left(M_{i}^{k} \kappa\left(M_{[j]}^{[l]}\right)\right)\left(\mathbf{A}_{k[l]}^{m[n]} * a\right), \\
& \left(a * \tilde{\mathbf{A}}_{i[j]}^{k[l]}\right) \kappa^{-1}\left(\kappa\left(\bar{M}_{k}^{m}\right) \bar{M}_{[l]}^{[n]}\right)=\kappa^{-1}\left(\kappa\left(\bar{M}_{i}^{k}\right) \bar{M}_{[j]}^{[l]}\right)\left(\tilde{\mathbf{A}}_{k[l]}^{m[n]} * a\right) .
\end{aligned}
$$

It is sufficient to prove this relation for the generators. We give here the proof only for Eq. (5.80), since the proof for Eq. (5.81) follows analogously. Taking $a=\kappa^{-1}\left(M_{t}^{s}\right)$, Eq. (5.80) is

$$
\begin{aligned}
& \mathbf{A}_{i[j]}^{k[l]}\left(\kappa^{-1}\left(M_{t}^{p}\right)\right) \kappa^{-1}\left(M_{p}^{s}\right) \kappa^{-1}\left(M_{k}^{m} \kappa\left(M_{[l]}^{[n]}\right)\right) \\
= & \kappa^{-1}\left(M_{i}^{k} \kappa\left(M_{[j]}^{[l]}\right)\right) \kappa^{-1}\left(M_{t}^{p}\right) \mathbf{A}_{k[l]}^{m[n]}\left(\kappa^{-1}\left(M_{p}^{s}\right)\right) .
\end{aligned}
$$

Using

$$
\mathbf{A}_{i[j]}^{k[l]}\left(\kappa^{-1}\left(M_{t}^{s}\right)\right)=F_{-[j]}^{[l]}\left(M_{s^{\prime}}^{s}\right) f_{+i}^{k}\left(M_{t}^{s^{\prime}}\right),
$$

and the $\hat{\mathbf{R}}$-matrix representation of the functionals $F$ and $f$, we find that Eq. (5.82) holds due to the fundamental relation of the quantum group (1.6). When 
$a=\kappa^{-1}\left(\bar{M}_{t}^{s}\right)$, the proof is parallel to the previous one and Eq. (5.80) holds due to Eq. (1.10).

Then using the relation Eq. (5.80), the coaction on the second term of Eq. (5.70) is calculated as

$$
\begin{aligned}
\Delta_{L}\left[\left(x * \mathbf{A}_{i[j]}^{k[l]}\right) \nabla_{k[l]}\right] & =\sum_{s}\left(a_{s} * \mathbf{A}_{i[j]}^{k[l]}\right) \kappa^{-1}\left(M_{k}^{k^{\prime}} \kappa\left(M_{[l]}^{\left[l^{\prime}\right]}\right)\right) \otimes x_{s} \nabla_{k^{\prime}\left[l^{\prime}\right]} \\
& =\sum_{s} \kappa^{-1}\left(M_{i}^{i^{\prime}} \kappa\left(M_{[j]}^{\left[j^{\prime}\right]}\right)\right)\left(\mathbf{A}_{i^{\prime}\left[j^{\prime}\right]}^{k^{\prime}\left[l^{\prime}\right]} * a_{s}\right) \otimes x_{s} \nabla_{k^{\prime}\left[l^{\prime}\right]} \\
& =\sum_{s} \kappa^{-1}\left(M_{i}^{i^{\prime}} \kappa\left(M_{[j]}^{\left[j^{\prime}\right]}\right)\right) a_{s} \otimes\left(x_{s} * \mathbf{A}_{i^{\prime}\left[j^{\prime}\right]}^{k^{\prime}\left[l^{\prime}\right]}\right) \nabla_{k^{\prime}\left[l^{\prime}\right]},
\end{aligned}
$$

where $\Delta_{L}(x)=\sum_{s}\left(a_{s} \otimes x_{s}\right)$ with $a_{s} \in \mathscr{A}$ and $x_{s} \in \mathscr{M}$. Equation (5.79) can be derived analogously by using Eq. (5.81). Therefore, we get the transformation of the commutators (5.78) and (5.79) from the definitions (5.76) and (5.77).

q.e.d.

This proves the covariance of the comutators given in Eqs. (5.70) and (5.71). Furthermore, Proposition 6 implies

$$
\begin{aligned}
& \Delta_{L}\left(\theta^{i[j]}\left[\nabla_{i[j]}, x\right]_{q}\right)=\sum_{s} a_{s} \otimes \theta^{i[j]}\left[\nabla_{i[j]}, x_{s}\right], \\
& \Delta_{L}\left(\bar{\theta}^{i[j]}\left[\tilde{\nabla}_{i[j]}, x\right]_{q}\right)=\sum_{s} a_{s} \otimes \bar{\theta}^{i[j]}\left[\tilde{\nabla}_{i[j]}, x_{s}\right],
\end{aligned}
$$

where $\Delta(x)=\sum_{s}\left(a_{s} \otimes x_{s}\right)$. This means if we use the derivative defined in Eqs. (5.74) and (5.75), i.e., if we define the derivative by

$$
\mathbf{d} x=\theta^{i[j]}\left[\nabla_{i[j]}, x\right]+\bar{\theta}^{i[j]}\left[\tilde{\nabla}_{i[j]}, x\right],
$$

then the derivative $\mathbf{d}$ satisfies the requirement of the covariance (5.57) and this shows the consistency of the identifications (5.68) and (5.69). Note that in our construction, the condition of the covariance (5.57) holds separately for $D$ as well as for $\bar{D}$.

Therefore, the $\nabla_{i[j]}$ and $\tilde{\nabla}_{i[j]}$ define comodules and the commutators in Eqs. (5.70) and (5.71) transform in a consistent way with the transformation properties of $\theta^{i[j]}$ and $\bar{\theta}^{i[j]}$, respectively ${ }^{3}$ and thus they are consistent with Eq. (5.57).

To complete the algebra containing $\nabla_{i[j]}$ and $\tilde{\nabla}_{i[j]}$, we must also define the commutation relation among them. This can be performed using the correspondence between $\nabla_{i[j]}\left(\tilde{\nabla}_{i[j]}\right)$ and the functionals $\chi_{i[j]}\left(\tilde{\gamma}_{i[j]}\right)$ given in Eq. (5.68) (resp. Eq. (5.69)).

\footnotetext{
${ }^{3}$ Recall that in our present notation the transformation rule of the basis $\theta^{i[j]}$ is

$$
\Delta_{L}\left(\theta^{i[j]}\right)=M_{\imath^{\prime}}^{i} \kappa\left(M_{\left[j^{\prime}\right]}^{[j]}\right) \otimes \theta^{i^{\prime}\left[j^{\prime}\right]},
$$
}

which is equivalent to Eq. (4.3) in ref. [CSWW] using the definitions (1.17) and (1.18) together with (1.14). Similarly for the basis $\bar{\theta}^{i[j]}$ in our present notation, the left-coaction is given by

$$
\Delta_{L}\left(\bar{\theta}^{\imath[J]}\right)=\kappa\left(\bar{M}_{i^{\prime}}^{\iota}\right) \bar{M}_{\left[\jmath^{\prime}\right]}^{[\jmath]} \otimes \bar{\theta}^{i^{\prime}\left[j^{\prime}\right]} .
$$


Acknowledgement. The authors would like to thank to Dr. M. Schlieker for helpful discussions about the dual Hopf algebra of the complex quantum group. They also thank Dr. P. Podles for discussions about the double group construction of the complex quantum group.

\section{Appendix}

We give here the definition of the various $\hat{\mathbf{R}}$-matrices. As we mentioned, we do not apply the index convention (1.17) and (1.18) for the $\hat{\mathbf{R}}$-matrix,

$$
\begin{aligned}
\hat{\mathbf{R}}_{[l] j}^{ \pm i[k]} & \equiv \varepsilon_{[l] s} \hat{\mathbf{R}}_{j t}^{\mp s i} \varepsilon^{t[k]}, \\
\hat{\mathbf{R}}_{l[j]}^{ \pm[i] k} & \equiv \varepsilon^{[i] t} \hat{\mathbf{R}}_{t l}^{\mp k s} \varepsilon_{s[j]}, \\
\hat{\mathbf{R}}_{[l][j]}^{ \pm[i][k]} & \equiv \varepsilon_{[l] t} \varepsilon_{[j] s} \hat{\mathbf{R}}_{m n}^{ \pm s t} \varepsilon^{n[i]} \varepsilon^{m[k]} .
\end{aligned}
$$

\section{References}

[Abe] Abe, E.: Hopf Algebras. Cambridge Tracts in Math., vol. 74, Cambridge Univ. Press, 1980

[CSSW] Carow-Watamura, U., Schlieker, M., Scholl, M., Watamura, S.:Z. Phys. C-Particles and Fields 48, 159-165 (1990); Int. J. of Mod. Phys. A6, 3081-3108 (1991)

[CSW] Carow-Watamura, U., Schlieker, M., Watamura, S.: Z. Phys. C-Particles and Fields 49, 439-446 (1991)

[CSWW] Carow-Watamura, U., Schlicker, M., Watamura, S., Weich, W.: Commun. Math. Physics 142, 605- 641 (1991)

[Dri] Drinfeld, V.G.: Quantum Groups, Proceedings of the International Congress of Mathematicians. Vol. 1, 798-820 (1986)

[DSWZ] Drabant, B., Schlieker, M., Weich, W., Zumino, B.: Complex Quantum Group and Their Quantum Enveloping algebras. MPI-PTh/91-75

[Jim] Jimbo, M.: Lett. Math. Phys. 10, 63-69 (1986)

[Jur] Jurco, B.: Lett. Math. Phys. 22, 177 (1991)

[Koor] Koornwinder, T.: Unpublished note (1990)

[LNRT] Lukierski, J., Nowicki, A., Ruegg, H., Tolstoy, V.N.: Phys. Lett. B264, 331 (1991)

[LNR] Lukierski, J., Nowicki, A., Ruegg, H.: Real Forms of Complex Quantum AntideSitter Algebra $\mathscr{U}_{q}\left(S_{p}(4, C)\right)$ and Their Contraction Schemes. Univ. of Geneve (1991) (Preprint)

[Manin] Manin, Yu.I.: Quantum groups and non-commutative geometry. Centre de Recherches Mathematiques, Université de Montreal (1988)

[MH] Müller-Hoissen, F.: GOET-TP 55/91 (1991)

[MNW] Masuda, T., Nakagami, Y., Watanabe, J.: K-Theory 4, 157-180, (1990)

[OSWZ] Ogievetsky, O, Schmidke, W.B., Wess, J., Zumino, B.: MPI-Ph/91-51 (1991)

[Pod1] Podleś, P.: Complex quantum groups and their real representations. RIMS-754 (1991)

[Pod2] Podleś, P.: Lett. Math. Phys. 18, 107-119, (1989)

[Pusz] Pusz, W., Woronowicz, S.L.: Rep. Math. Phys. 27, 231-257 (1989)

[PW] Podleś, P., Woronowicz, S.L.: Commun. Math. Phys. 130, 381-431 (1990)

[Rosso] Rosso, M.: Duke Math. J. 61, 11-40 (1990)

[RTF] Reshetikhin, N.Yu., Takhtadzhyan, L.A., Faddeev, L.D.: Algebra and Analysis 1, 178-206 (1989); Leningrad Math. J. 1, 193-225 (1990)

[Schm] Schmüdgen, K.: Covariant differential calculi on quantum spaces. Leipzig preprint [Stach] Stachura, P.: Bicovariant differential calculi on $S_{\mu} U(2)$, to appear in Lett. Math. Phys.

[SWZ] Schmidke, W.B., Wess, J., Zumino, B.: Preprint MPI-Ph/91-15 (1991) 
[Weich] Weich, W.: Ph.D. Thesis: Die Quantengruppe $S U_{q}(2)$-kovariante Differentialrechnung und ein quantensysmmetrisches quantenmechanisches Modell. Karlsruhe University, November 1990

[Wess] Wess, J.: Talk given in 300-Jahrfeier der Mathematischen Gesellschaft in Hamburg, Hamburg 1990

[Wor1] Woronowicz, S.L.: Commun. Math. Phys. 111, 613-665 (1987)

[Wor2] Woronowicz, S.L.: Commun. Math. Phys. 122, 122-170 (1989)

[WZ] Wess, J., Zumino, B.: Nucl. Phys. B (Proc. Suppl.) 18B, 302 (1990)

[Zumino] Zumino, B.: Talk given in Recent Advances in Field Theories, Annecy meeting in honour of R. Stora, 1990

Communicated by H. Araki 